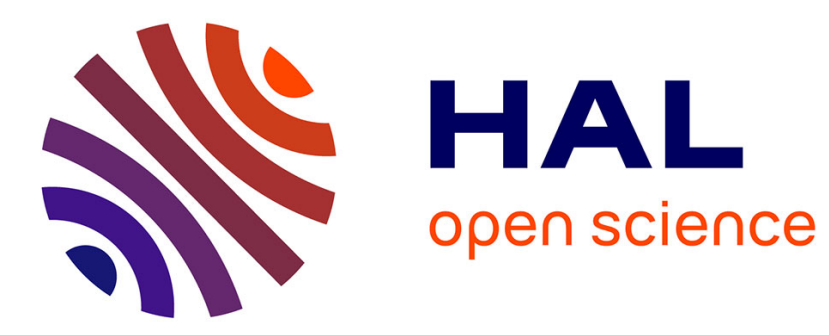

\title{
À propos de CATVSSA
}

Colette Bémont

\section{To cite this version:}

Colette Bémont. À propos de CATVSSA. Gallia - Fouilles et monuments archéologiques en France métropolitaine, 1973, 31 (1), pp.81-122. 10.3406/galia.1973.2626 . hal-01934111

\section{HAL Id: hal-01934111 \\ https://hal.science/hal-01934111}

Submitted on 11 Mar 2020

HAL is a multi-disciplinary open access archive for the deposit and dissemination of scientific research documents, whether they are published or not. The documents may come from teaching and research institutions in France or abroad, or from public or private research centers.
L'archive ouverte pluridisciplinaire HAL, est destinée au dépôt et à la diffusion de documents scientifiques de niveau recherche, publiés ou non, émanant des établissements d'enseignement et de recherche français ou étrangers, des laboratoires publics ou privés.

\section{(이)(\$)}

Distributed under a Creative Commons Attribution - NonCommercial - NoDerivatives| 4.0 


\title{
A PROPOS DE CATVSSA
}

\author{
par Colette BEMONT
}

Malqué une série de travaux de qualité, dont le plus important, à ce jour, est le Central Gaulish Pollers ${ }^{1}$, l'étude des atcliers du $\mathrm{II}^{\mathrm{e}}$ siècle, à Lezoux, comporte de nombreuses lacunes. L'inventaire des moules de la collection Plicque, entrepris depuis peu au Nusée des Antiquités nationales de Saint-Germain-en-laye, ma permis de regrouper, parmi les exemplaires signés, un ensemble de pièces portant, manuscrit, le nom CATYSSA ou CATYSA, plus ou moins largement conservé. Lne enquète préalable sur la place réservée, dans les publications, à ce fabricant de matrices a montré que sa signature était bien connue par les relevés de .J. Déchelette (à partir, surtout, de cette même collection Plicque $)^{2}$-- complétés par quelques exemplaires nouveaux, issus de collections anglaises ${ }^{3}$ ou de récentes fouilles à Lezoux ${ }^{4}$-, mais que les déeors restaient pour la plupart à lécouvrir. L'étude de ce matériel, réduit mais d'attribution assurée, avait done toutes chances d'apporter quelques nouveautés. Je plus, G. Simpson et J. A. Stanfield avaient distingué, au moins provisoirement, d'après le type de signature surtout (manuscrite dans un cas, estampillée dans l'autre), deux potiers de ce nom. Lit il importait de voir dans quelle mesure une connaissance un peu plus large du répertoire décoratif confirmait le bien fondé de cette distinction ou amenait à en nuancer l'interprétation. Enfin, l'examen de ces quelques fragments a fait apparaître, pour la seule série inscrite, une richesse dans l'invention ornementale et une diversité dans l'orthographe, ou la forme, des signatures qui conduisent à s'interroger sur son homogénéité.

J. Déchelette signale, dans la collection Plicque, quatre échantillons : "1. Frag. de moule. leécor à médaillons. Deux zones. CATVSAs MANV, graffite;2. Frag. de moule. CATVSSA, graffite ; 3. Frag. de moule 37. CATVS. ., graffite ; 4. Frag. de moule 37, . TVSSA MA . , graffite ». L'inventaire des moules ou fragments du musée fait apparaitre, dès l'abord. une lacune : l'absence du graffite [...TVSSA MA [...] En revanche, la signature CATVSAs MANV (fir. 11, I, i) et l'énumération de quelques poinçons décoratifs permettent de reconnaitre un moule à trois zones ornées (et non deux).

1 J. A. Stanflede el G. Sumpos, Oxford, 1958 abrege en S.-S. .

2 Vases céramiques ornés de la (iaule romaine, Paris, 1904, I, p. 259-260, no 47.

3 S.-S., pl. 152, 1 vase signé.

4 Plusieurs exemplaires mont eti aimablement signales par H. Hartley, comme appartenant aux collections du musere on comme provenant des fouilles de la route de Maringues. La photographie de l'un deux figure au no 3 du Bull. du Com. archiol. de L.ezour 1970, p. 9.

5) Op. cil., p. 256 .

6 Intradecorative, sur deux lignes, avant cuisson. 
dont il subsiste plusieurs morceaux ${ }^{7}$ (fig. 2). Pour le araffite CATVSSA, l'hésitation est possible : trois matrices 37 portent, en effet, la même inscription (fig. 11, I, 1, a, b, d) et Jéchelette n'a signalé aucun élément de décor, qui autoriserail a choisir entre deux frises de style libre et un rinceau a bouches coupées ${ }^{9}$. Cependant le fac-similé publié ressemble à l'une de nos signatures (fig. 11, I, d) beaucoup plus qu'aux deux autres : on peut done penser que le graffite est celui d'un grand bol. maintenant remonté à partir de plusieurs morceaux et orné d'animaux, sur un fond semé de petites feuilles ${ }^{10}$. Il existe, enfin, dans la collection, un fragment de moule 37 porlant, sous une frise de dauphins el. de tritons, une suite de caracteres diversement conservés qu'on ne peut lire que . . G.ATV: ... (lig. 11, I, g $)^{11}$. Watheureusement, aucun fac-similé n'accompagne le no 3 , ce qui interdit toute identification certaine, d'autant plus qu'un seul motif décoratif est signalé par Iéchelette et qu'il paraît étranger à notre décor. d l'examen. Loutefois, ces réserves ne sont pas délerminantes; d'autres exemples (ainsi C.ITVSAs MANV) montrent que, dans son répertoire épigraphique, J. Iéchelette. n'a retenu, le plus souvent, qu'une partie des décors figurés sur les vases signés, même quand les autres motifs apparaissent, par ailleurs, dans son inventaire des poinçons ${ }^{12}$. I'autre part, il est, vai que le sujet reconnu est différent de celui que l'on distingue sur notre fragment de moule, mais il ne s'ensuit pas nécessairement qu'il ne s'agisse pas de la même pièce car l'état de la matrice près de la cassure n'exclut pas la possibilité d'une erreur. La représentation réelle : un membre inférieur -- pisciforme et enroulé verticalement -- de triton (fign. 6), n'est pas sensiblement différente. dans sa forme générale, du dauphin enroulé autour d'un axe (une rame? qu'a va Iéchelette ${ }^{13}$. I)e plus, la fracture du moule, toul en faisant disparaitre la quasi tolalité du reste du triton, a laissé subsister un fragment du feston d'où, au niveau des hanches, sortent les membres monstrueux, et la confusion est aisée entre ces dentelures et la collerelle qui indique, traditionnellement, la démarcation entre la tête et le corps des dauphins.

Ainsi, nous avons cing moules (fig. 1) dont trois appartiennent sùrement, ou peuvent appartenir, au rroupe connu de Ibechelette : trois matrices sont décorés dans le style libre --.- chasses ou animaux et monstres marins -.. - la quatrieme est ornée d'un rinceau habité, la cinquième, d'un rinceau et de deux zones de métopes. Quatre ont conservé une signature complete ef le nom apparait une fois sous la forme CATTSA (ou CATYSAS), trois fois sous la forme CATVSSA. Ia derniere inscription est mutilée et son ériture, une sorte de capitale peu soignée, la distingue des autres. Véanmoins la seule lecture possible oblige à reconnaitre le groupe liATVS et le seul nom, actuellement signalé, qui comporte. ces deux syllabes, est CATYSSA, on CATYSA. Il convient, semble-t-il, d'ajouter à cette série un petit fragment de moule 37, qui porte, sous un déor de métopes, les restes d'une inscription (fig. 3,3;11, I, c). Celle-ci nous fournit la fin d'un mot : l'espacement des caractères est régulier et un petit trait horizontal au bord de la cassure révèle l'existence au moins d'une lettre avant la première qui subsiste en entier. Le fragment fait apparaître, en outre, les traces d'un essai de destruction : des stries horizontales barrant les lettres

7 MAN 66381. Le remontage des fragments permet de constituer deux consembles continus, courrant plus de Ia moitié des deux zones inferieures du décor.

8 MAX 65993 et P.M. 2 MAX ot P.M. renvoient respectivement aux inventaires ancirns et en cours de la coll. Plicquei.

9 MAX $663 \times 0$.

10 MAX 65993. L'absence d'informations sur la frise peut s'expliquer aisement, si la lecture a eté faite avant la restauration du moule : les cassures séparent le fond 'signé du decor.

11 P.M. 3. Signature infradecorative, avant cuisson.

12 Trois poincons de la matrice 66381 ont été retenus, sur les seize que comporte le répertoire et le plus grand d'entre cux, la Vénus, a eté omis.

13 Op. cil., p. 259, renvoyant au $n^{\circ} 1060$ de l'inventaire des poinçons. 

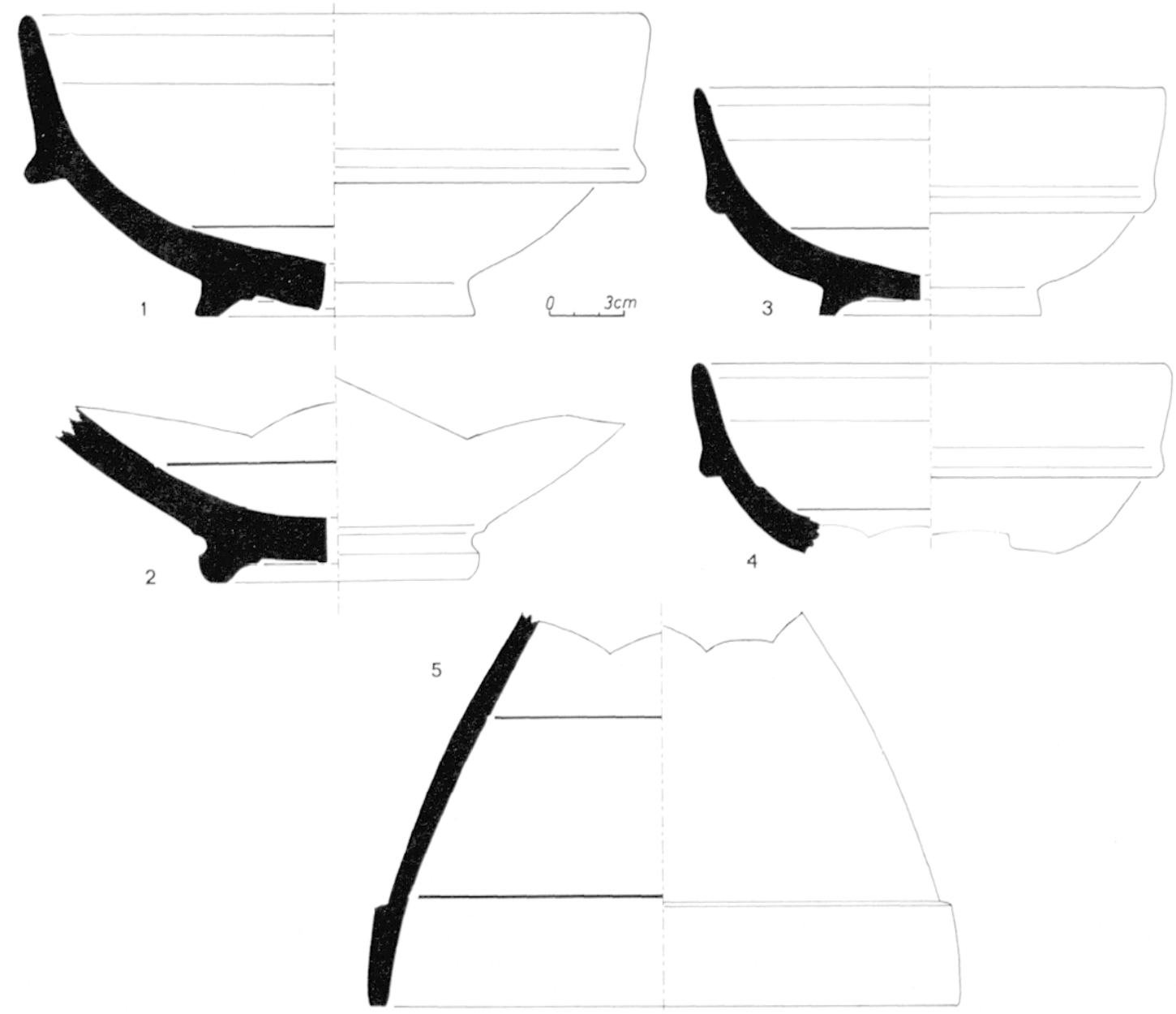

1 Moules de cATVSSA an Musee des Antiquités nationales : profils et coupes. 1 : D. 37, 659993; 2: 1), 37, P..Y. 2 ; 3: D. $37,663 \times 0 ; 4:$ D. 37 , P... 3 ; is : lagene, $66.3 \times 1$.

et les ayant partiellement endommagées aprés cuisson ${ }^{14}$. On lit sans difficulté a, puis, après trois hastes tronquées et courbées, en has, vers la droite, s, s, a. Les deux derniers signes, avant les $s$, ne peuvent correspondre, en l'absence de toute autre trace ${ }^{15}$ et compte tenu de leurs courbure et direction respectives qu'au reste d'un $u^{16}$. Nous lisons donc .a.ussa. L'hésitation est, dès lors, possible entre TALVSSA - attesté par un graffite de Roanne ${ }^{17}$ - et CATVSSA, mais la seconde hypothèse parait la meilleure. En effet, la trace horizontale avant l'a est très compatible avec le ductus d'un $c$, moins conciliable avec celui du $l$ (présentant, le plus souvent, une courbure moins prolongée ${ }^{18}$ ). I'autre

14 P.M. 1. Cet accident niest pas unique : par exemple, un lesson inédit de la mème collection porte une signature barrée dans le moule estampille intradecorative de BVTKIO ; un fragment de matrice conserve un graflite (DOCI...) volontairement rạe.

15 Ine verification a la loupe binoculaire ma permis de voir que des traces verticales, apparentes sur le frottis, résultaient d'erafhures postérieures a la cuisson : il n'y a donc pas lien de retenir l'hypothese d'un aça a laquelle mon ami G. H. Rogers avait, un moment, songé.

16 Je remercie R. Marichal qui a bien voulu examiner le tesson et confirmer la vraisemblance de cetle restitution.

17 J. Dёснететте, op. cit., I, p. 301, no 188.

I8 Le $T$ de TAIVSSA, dans la signature connue, se recourbe bizarrement en formant un angle tres aigu. 
part, le sillon qui, après l'a, raie la surface a la partie supérieure du corps des lettres, explique sans mal la disparition de la barre du $l$. In $l$ est plus difficile a restituer, car la forme qu'il prend dans certaines signatures contemporaines, à Lezoux : ${ }^{19}$, est peu en rapport avee l'arrèt brutal de la haste vers le bas balors que cette partie du moule n'est pas érodée ${ }^{20}$ même si l'on admet, par ailleurs, que la lettre plus ou moins haute.n'avait pas de raison de laisser obligatoirement une trace au-dessus des ratures. Ln $\alpha$, forme également possible ${ }^{21}$. n'est pas plus satisfaisant : on ne s'explique pas la disparition totale de la grande haste après l'a, alors que l'usure produite par les traits horizontaux a laissé subsister des fragments de tous les autres tracés verticaux. La restitution C. ATYSSA est done, pour res raisons, la plus plausible. En outre, il convient de souligner que la signature connue de TALVSSA figure sur la surface exlérieure d'un moule, a la différence des graffites de CATVSSA qui sont tous infra- ou intradécoratifs. Enfin, nous verrons plus loin que l'attribution du fragment au second ne souleve pas de difficultes, lorsqu'on examine le répertoire décoratif.

L“inventaire du matériel signé conduit done a rassembler une série de matrices portant deux formes, ou deux noms, voisins, sans être identiques : CATYSSA et CATTSAS. Y-at-lil des raisons philologiques de distinguer, par leur nom, deux potiers? Qu'il ait existé concurremment des formes avere un et deux $s$, nous an avons, apparemment, lat preuve par une signature sur moule 37 récemment signalée a Lazoux : . . ATVSA $11{ }^{22}$ et attribuée sans hésitation à CATVSA. Et l'on considere, d'ordinaire, l'alternance - $s-$ - $s s$ romme une variation de la graphie d'un même mot ${ }^{23}$. En revanche la désinener de catTSAS a suscité, depuis la déenuverte du moule, des explications diverses : on a imagine. pour justifier le s final, de le dissocier de CATVSA ot d'en faire l'abreviation de seruus ${ }^{24}$, mais il faut remarquer que cethe signature serait lat seule connue de ce genre et l'on souhaiterait, pour en arcepter l'hypothese aver moins d'hésitations, que d'autres exemples romparables la confirment ; de plus, l'absence d'intervalle entre l'a cet le s. alors que la place ne manque pas pour inscrire la lettre un peu plus loin, invite plutôt à considérer CiTTSAS comme un seul mot ${ }^{26}$. J. Wéchelette, écartant déjà cette supposition, penchait en faveur d'une correction maladroite et du rétablissement, hors de sa place, du s oublié de ciATYSSA ${ }^{27}$. L'équivalence, bien admise, de -s- et -ss- et, en outre, l'existence, sur place, l'une signature infradécorative [c]ATVSA rendent cette supposition, un peu hasardée, encore plus fragile. CATVSAS ne peut-il être simplement une forme correcte de nominatif ou de génitif?

19 Cif., en particuliur, Jes simalures : PLAVTIXIMAX 66021, 66053, 66062, 66064 entre antres .

20) A la Joupe, la surface du moule paraît, à cel endroit, intacte ef l'exlremite de la haste, bien conservee.

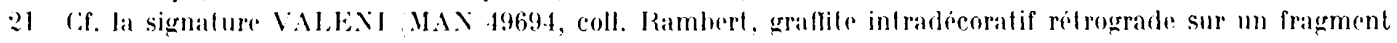
de bol I). 37 .

22 lif. n. 4, exemplaire photographic.

23 Pour Jes graphies avec un ou deux s, ef. G. JorTix, La langue gauloise, Paris, 1920, p. 11: : (:1TVSo/ CATVSOS.

2.4 C:est Jinterpretalion suggere par 0. Bohn (C.I.I. XIII, 10011, 5l .

25 On a proposé le plus souvent dinterproter l'estampille du sud de la Gaule : GERMAXI SBR, comme GEIMANI SElaus o Je connais, pour ma part, à Arles, une mardue de la Graufesenque - complete dans sa derniere partie - : F. TORXI.S, dont l'explication est problematique. Aucune de ces signatures, au demeurant isolees, ne contient $M \mathrm{ANV}$.

26 La place etait suffisante, en tout cas, pour ponctuer.

27 Op. cil., I, p. 151, n. 2. 
I). Ellis Evans signale quelques nominatifs en -as- principalement des ethniques 28 -. et une série de noms masculins en - $a$, dont certains ont un génitif en -as ${ }^{29}$. Il n'y aurait donc pas d'impossibilité formelle à ce que CATYSAS füt normal, en particulier comme génitif ${ }^{30}$, et se justifiât de la sorte aviant manu. Il n'en demeure pas moins que la disparité pose, en soi, un problème: quelle que soit l'éventualité retenue, la forme en -as concurrence la forme en -a comme nominatif ${ }^{31}$, et l'on a deux sollécismes, ou le génitif correct caTVSAS alterne, devant manu, avec le nominatif fautif ${ }^{32}$. Il ne semble donc pas quil y ait un obstacle théorique à ce que CATTSSA, CATTSA et CATYSAS soient des formes lorthographiques ou grammaticales) différentes d'un même nom : la difficulté réside plutôt dans l'emploi conjoint qu'on en a fait et dans la signification qüil faut donner a cette concurrence. Liexamen du style des différents décors nous fournira peut-être un moyen de comprendre ces différences en suggérant des groupements, et, si ce n'est pas possible, du moins pouronsnous espérer tirer de l'enquête des présomptions solides en faveur de l'homogrenceité de l'ensemble du matériel ou à l'encontre de cette hypothèse.

Ce petit groupe de moules frappe d'abord l'attention par la variété des types de décors représentés : les uns, a base géometrique, présentent une division régulière at apparente, les autres relevent du style dit "libre ", au sens où les motifs figurés semblent se poursuivre sans ordre immédiatement visible sur le fond lisse de la matrice ${ }^{33}$. A l'intéricur mème des catégories principales des nuances apparaissent : les frises géométriques s'organisent tantôt en métopes ${ }^{34}$, tantôt en rinceau ${ }^{35}$, ou encore présentent une combinaison des deux systèmes ${ }^{36}$. Il n'y a pas lieu, en effet, de distinguer, en l'occurrence, le rinceau de tout autre procédé de partage de la surface : nous n'avons plus sous les yeux lo souple déroulement d'une guirlande tracée à main levée, mais le résultat d'un procédé constamment employé dans d'abondantes séries de même origine : l'impression mécanique du même poinçon décoratif. Ie plus, le poinçon, lui-même, n'est plus, comme il advenait souvent pour les frises de l'école d'Aleius ou sur les D. 29 de la Graufesenque, un motif complexe orné de feuilles, de vrilles, ou de fleurs, mais une forme géométrique sèche : un simple demi-cercle, coupé et meublé ensuite comme on le ferait de n'importe quelle métope ${ }^{37}$. Nous n'arons donc là qu'un moyen élégant, mais automatique et monotone, de ménager régulièrement des espaces à orner.

Les décors libres relevent, eux aussi, d'inspirations diverses : l'un des moules est réservé à des sujets marins, les deux autres à des ménageries - saurages ou domestiques -. auxquelles se mêle, sur un fragment, un chasseur. Voyons d'abord dans quelle mesure

28 Gaulish personal Names, Oxford, 1967, p. 58, 305, 306 'n. 1 et 2, ,369. Je remercie I. Flenriot de l'ajde qu'il a bien voulu mapporter dans cette recherche.

29 Ibil., p. 56, n. 1 (noms masculins en -a ; p. 121 (génitifs en -as.

30 En labsence d'autres exemples, incontestables, il ne sagit evidemment que d'une hypothese.

31 On considere alors qu'on a perdu de vue le génetif determinant qui logiquement precedait MA.TV. Cétle hesitation paraît déjà se manifester au I $^{\mathrm{er}}$ siècle, dans le Sud, devant OFFICINA on MANT.

32 Aucun autre CATVSAS, en quelque fonction que ce soit, nest encore signale.

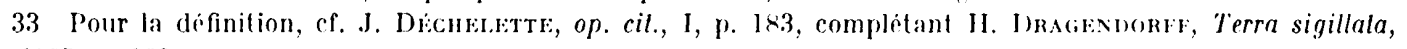
Bonn, 1895, p. 132.

34 P...I. 1.

$35 \mathrm{MAN} 66380$.

36 \А. 66.381 .

37 Ce procédé paraît commun à la plupart des decorateurs du ule siecle, à Ifzoux ce. les exemples fournis par S.-S. pour X-2, RAXTO, MEUETVS-IANTO, SIIVIO, IOENAIIS, DONNAVIVS, I.IBERTVS, BVTRIO, SACFR, ATTIANS, DRVSVS, PATERNVS, CIICIRO, DOECCVS, AVENTHYS, GIXXAMVS. 


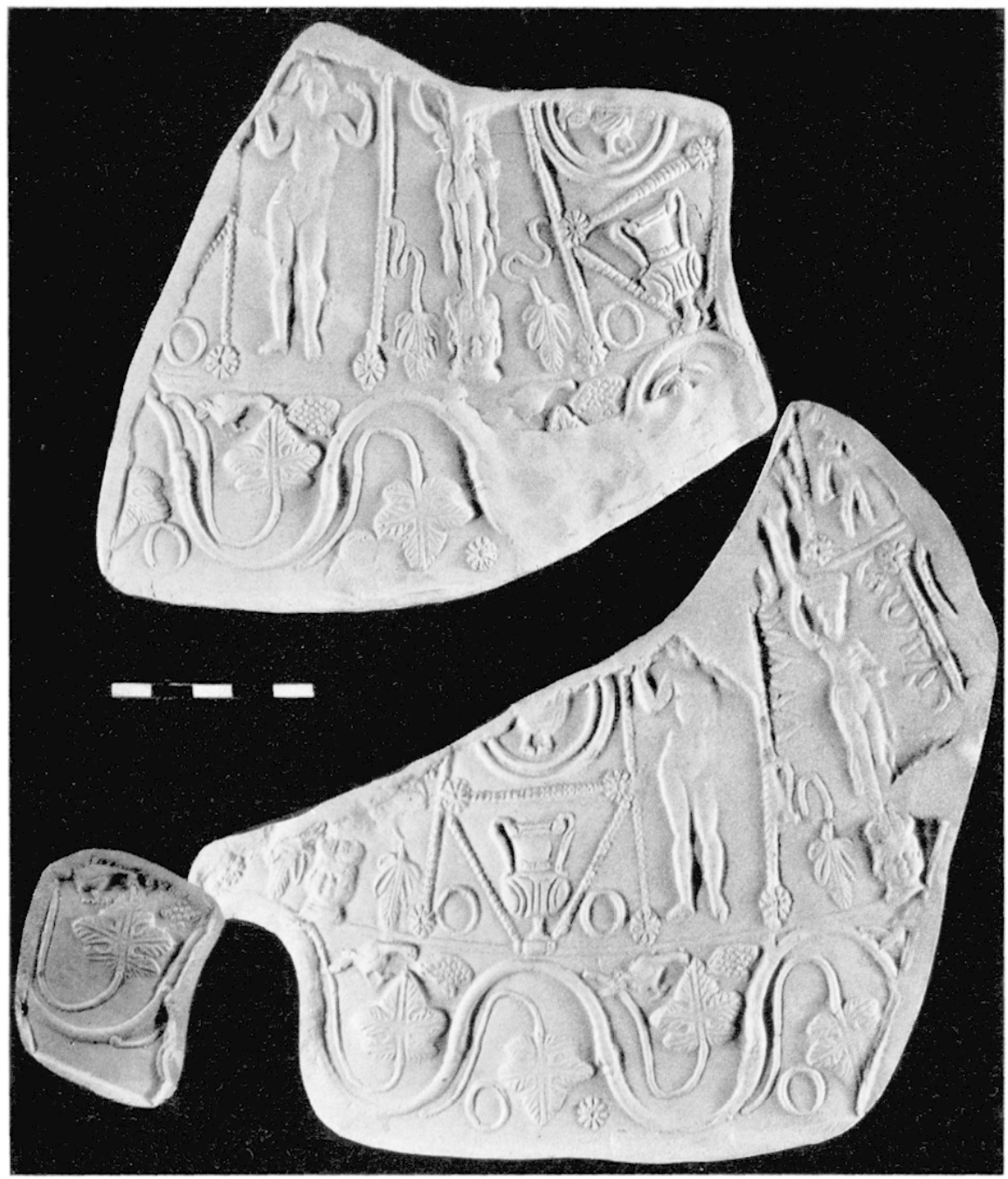

2 Moule do CATVSSA : empreinte des fragments de la valve de lagène 66381 ).

il y a lieu de séparer du groupe, provisoirement supposé homogène, le moule signé CiATISAS MANT, en examinant le décor dans son détail. Puis nous chercherons à définir en eux-mèmes les principes, semblables ou divers, qui règlent l'ornementation de nos moules en les comparant aux techniques d'autres ateliers.

La première des matriers, signée CATVSAS MANV dans le décor, se distingue au milieu du groupe non seulement par son inscription, mais encore par sa forme (fig. 1, 5). J. Déchelette s'était abstenu, sur ce point, de toute identification, mais le Corpus des inscriplions lalines se fait l'écho d'une inexplicable erreur : il reconnait en cette pièce un moule 37, il s'agit, en fait d'une matrice de lagène. La confusion ne se justifie pas par la forme de l'objet ... tronconique et ressemblant plutòt à un très gros gobelet - - et elle est d'autant moins concevable que le diametre le plus large, au bord, se trouve vers le bas de la pièce. Car l'orientation de tout le décor montre que nous avons entre les mains une valve supéricure. Fore est de supposer que l'auteur de cette bévue a probablement complété une lacune de son information, sans voir les tessons et par simple analogie avec les exemplaires 
signés CATYSSA ${ }^{38}$. Il reste de la matrice deux groupes de fragments, plus ou moins largement jointifs (fig. 2). L'un et l'autre portent des décors identiques, pour les deux zones qui leur sont communes, le plus grand conscrve, en outre, une petite partie d'une troisième zone.

Le répertoire décoratif comprend un assez petit nombre de sujets : quatre personnages, deux oiseaux. trois éléments végétaux, un vase, six poinçons géométriques et accessoires (fig. 10, al', a2, b1, b2, n1, o1', 12, C1, E3, E6, F1. (i2, II1, .11, R1. T1). La répartition des motifs s'opère de façon en partie exclusive : la zone inférieure, occupée par le rinceau. ne comporte que des éléments géométriques et végétaux et un oiseau (figr. 10. Fi), F1, R1, C1, (j2, . W1, n1). La conception du décor présente une certaine unité, car le rinceau, ramené à son origine purement végrétale, s'organise de façon naturelle, sinon réaliste. selon un thème : la fiquration d'un pampre habité d'oiseaux. Les éléments adventices : une rosette et un petit cercle (fir. 10, Ci. B:3. destinés à meubler les vides, n'ont de justification que décorative, mais leur taille et leur forme leur permettent de s'intégrer sans difficulté à la frise. I'autre part leur présence contribue discretement. mais incontestablement, a atténuer la disparate entre le rinceau et les métopes, puisqu'ils se retrouvent, bien visibles, dans l'un et l'autre reqistres. Les zones supérieures, divisées en panneaux par des cordons perlés ${ }^{39}$ accusent, comme à plaisir, le caractère artificiel el composite de ce style. Des poinçons divers et disproportionnés sont répartis sur deux registres de hauteurs vraisemblablement différentes. Il reste, de celui du haut, la plus grande partie d'un petit gladiateur (fig. 10, a2) el un fragment d'un personnage, connu, par des exemples conplets, comme cuirassé et vêtu d'un manteau ${ }^{40}$ (figr. 10. al, al'). Yous noterons la différence de module des deux éléments de décor et le fait que l'homme cuirassé déborde légrerement. sur le registre inférieur : sans doute faut-il voir là la preuve que la hauteur de la métope suffisait à peine à le contenir, car l'organisation par ailleurs soignée n'incite pas à croire à une négligence. La seconde série révèle un souci de diversité, compte tenu de l'hétérogénéité des trois panneaux que nous y voyons juxtaposés. L'un, très simple el un peu étriqué, présente de face, sur un fond uni, une grande Vénus nue, hanchée, soulevant dans ses mains levées de part et d'autre de la tête deux mèches de sa chevelure dénouée (fig. 10, b1). Le second contient une petite canéphore, également de face, à demi voilée de draperies légères, debout sur un piédestal constitué par une grosse tête, apparemment léminine (fig. 10, b2), le fond du panneau est meublé, de chaque còté de ce socle, par une feuille composée, rattachée aux cordons verticaux par une tige sinueuse (fig. 10, (62). Ce procédé de remplissage relève d'une technique chère surtout à l'époque flavienne, mais il s'est perpétué à Lezoux, au moins jusqu'iu milieu du II siècle gràce à quelques potiers, tel CaAsVRIVs. La troisième métope se caractérise par sa géométrisation; coupée, en son milieu. par un cordon horizontal, elle comporte, il est vrai, deux motifs figurés : un coq et un canthare (fig. 10). ol' et 'T1), mais sévèrement enfermés dans des cadres rigides el factices. L'oiseau est inclus dans un médaillon circulaire (fig. 10, E6), le vase, inscrit dans l'un des trois triangles déterminés, à l'intérieur du demipanneau, par deux cordons obliques. Le décor est complété par deux pelits cercles, de part et d'autre du pied du vase. Force est, au moins, de constater la recherche dont témoigne un tel souci de variété dans l'organisation de chaque métope.

Tous les personnages ou animaux sont attestés par F. Oswald, certains, connus dans l'inventaire de Déchelette ${ }^{41}$. Quelques-uns ressortissent au répertoire de LIBLRTVS-BVTTRI() : I'homme cuirassé, le gladiateur, la colombe (fig. 10. al', a2, n1), et sont, ensuite, utilisés continument jusqu'a la fin du siècle. I'autres : la Vénus et la canéphore, n'étaient connus. d'après les catalogues, que dans la seconde moitié du siècle. mais l'inventaire de moules el de vases inédits me permet maintenant.

38 Pour le type de la lagine, cf. infra, p. 114 a 122.

39 Les séparations entre les registres sont constituees par des traits pleins.

40 F. OsWath, Index of Figure-types on Terra sigillata, liverpool, 1936-1937 iabrige O. : respectivement

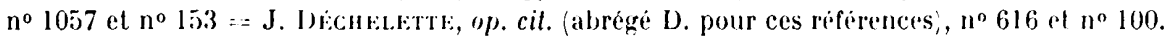

4 Vénus: $0.277 \ldots$ D. 172 ; canéphore : $0.1197 \mathrm{~A}$, cf. D. $199 ;$ colombe : $0.2234 ;$ coq : $0.2344 \mathrm{~A}=\mathrm{D} .1023$; vase: D. 1073 . 

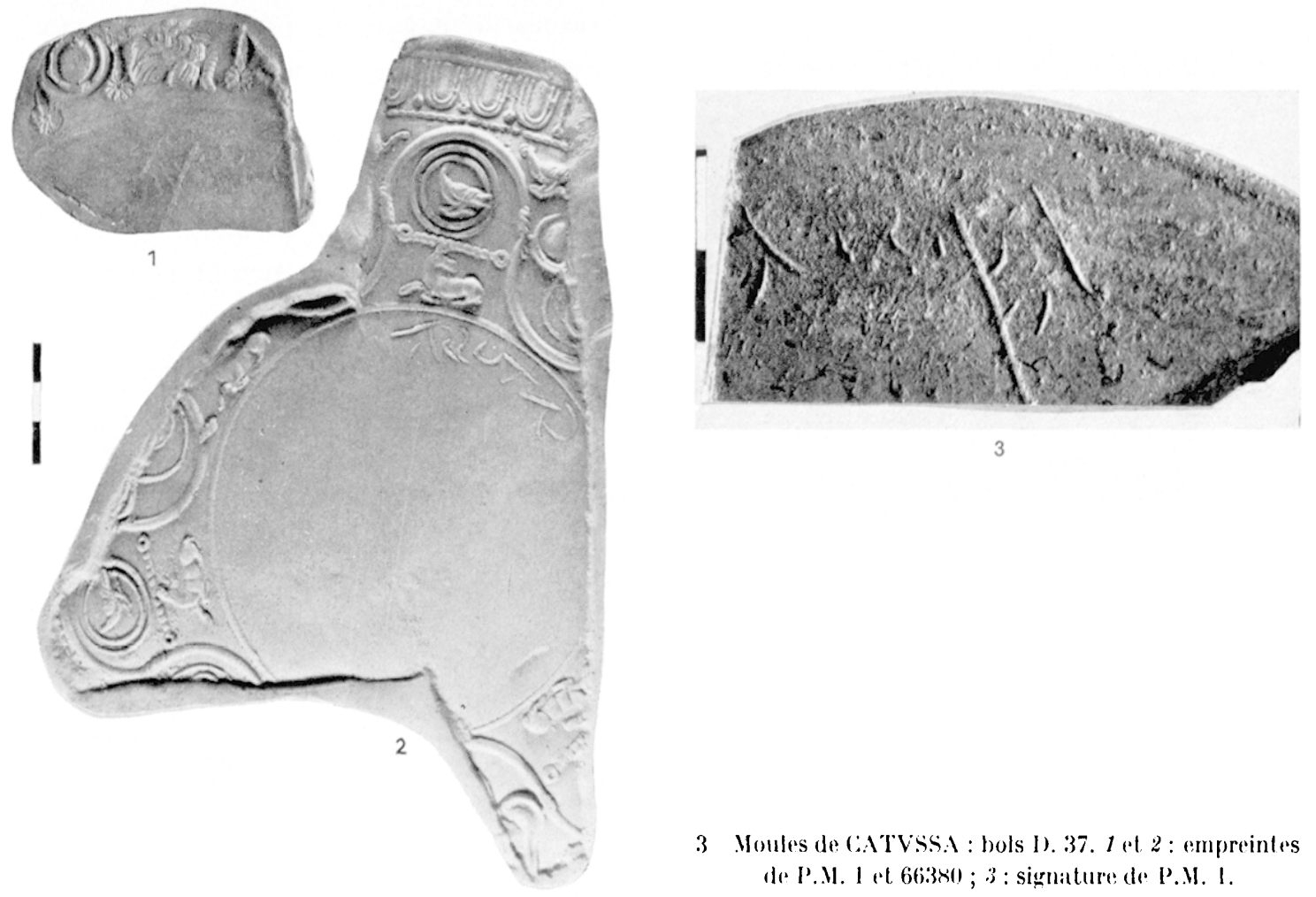

3 Noules de (CATVSSA : bols I). 37. 1 et 2 : empreintes de P...I. 1 (et $663300 ; 3:$ signature de P...I. I.

d'en rattacher l'origine au mème répertoire ${ }^{42}$. Le coq. enfin. attribué d'abord a BVTRIO), est ensuite

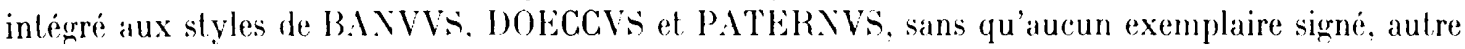
que ceux de CalTVSSA, soit signalé. Les décors secondaires, lorsqu'ils ont été retenus ${ }^{43}$ et reconnus par (i. B. Rogers ${ }^{44}$ chez des potiers datés, se prêtent à la mème localisation chronologrique (entre le deuxième el le quatrième quart du ${ }_{I I}$ e sièclej : le cordon perlé. utilisé d'abord par l'atelier de OVINTIIIANVS, est altesté sur des exemplaires signés jusqu'aux potiers de la dernière génération ${ }^{45}$; la feuille de vigne, la grappe, le vase sont employés par des artisans de la fin du siècle ${ }^{46}$; la petite feuille et la rosette. attestées chacune dans un atelier anonyme ${ }^{47}$ n'offrent que peu de ressources; le noud (figr. 10, R1), enfin, paraît propre à CATTVSsid.

42 La Vénus figure sur un moule D) 30 MAN $661 \mathrm{kx}$ de la coll. Plicque dans le style de LIBERTTS et portant, en bordure, l'ove circulaire pointé de ce potier. La canéphore, connue déja par un modele identique, mais réduit et

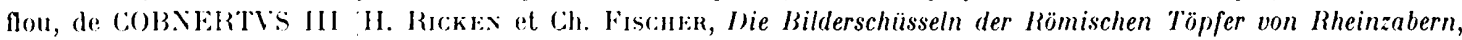
.Materialen zur rümisch-germanischen Keramik, H. 7, Bonn, 1963, p. 11-42: .1. 5i), apparait, parfaitement nette of semblable au poinçon de CATVSSA, sur un fragment anepigraphe de BVTKIC (MAN 49704, boh D. 37, et sur un moule 1). 37 .llN 66.tsx signe de LIBERTYS.

13 Jans son inventaire des poincons secondaires, (x. B. Rogers neglige dilibriment, comme trop peu significatifs et sujets a trop de variations de taille, les formes purement gémemitriques : cercles, demi-cercles, ou les sujets peu différenciés, tels les tortillons.

4 Poleries sigillies de la Giaule centrale. I : Les molifs non figurés. Je remercie tris vivement G. B. Rogers de m'avoir donné acces à ce tres important travail en cours de publication, auruel je me permettrai de renvoyer, avec l'accord de l'auteur.

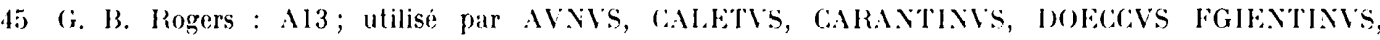
FLORIANVS, IVILICVS, IVIIINVS, MARCVS, RENTO, SISSVS, TALVSSA, TITIVS.

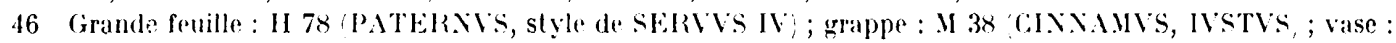

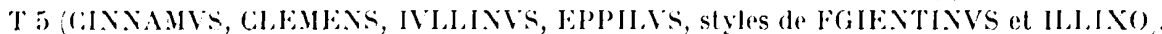

47 Rosette : : : 214 ; feuille : (2201. 
Le récor de la lagène, par les types d'ornementation de ses différentes zones, s'apparente, à la fois, all petit fragment signé A t ISS et conservant quelques métopes et au hol couvert d'un grand rinceau (fig. 1, 3).

Le prenier (fig. 3. I et 3. Lries exigu, garde les traces de trois panneaux : l'un renferme un fragrment de motif que je n'ai pu reconnaître, l'autre un masque de Pan (fig. 10, c1), de profil, disposé horizontalement. le troisieme contient un petit double-cercle (fig. 10. E4 Les cordons, tris écrasés, sont difficilement identifiables : on voil surtout qu iils étaient perlés. La rosette, imprimée a la base de chacun d'eux, est celle de la lagine. le seul élément nouveau, vraiment notable : le masque. est un motif bien connu. Attesté avant 150) dans l'atelier de DOCILIs et QVINTILIANVS, il se maintient, selon Oswald. jusqu'à la fin du sircle. Toutefois, il y aurait lieu de distinguer la permanence du motif de l'usiage commun d'un poinçon : il existe j'ai pu le constater sur des rases el des moules, plusieurs matrices semblables mais différant par la taille ou des détails ${ }^{18}$.

Le rinceau (fig. 3, 2), composé d'une suite de doubles-boucles, est, dans ses parties conservées, alternativement plein el coupé. Les boucles pleines, ouvertes vers le haut, sont occupées, au moins, par une feuille cordiforme (fig. 10. J1) et l'une. mieux conservée, contient en outre un oiseau (figr. 10, n2) ; les boucles coupées, ouvertes vers le bas, sont habitées par des animaux : un oiseau it gauche et palinscope (le mème que précédemment; une biche couchée, égatement à gauche et palinscope (fig. 10, g2). Le décorateur s'écarte, en la circonstance. du partie naturaliste adopté pour la lagène, en traitant une partie de la frise comme une suite de métopes coupées, et souligne le caractère géométrique de son projet en enfermant, de surcroît, l'oiseau dans un double cercle (fig. 10, Ej). Autre différence avec la valve : si l'arceau utilisé est bien le mème par sa forme el ses dimensions le raccord entre deux demi-cercles conséculifs est dissimulé par un tortillon (fig. 10, 1\%1), au lieu d'un norud.

Iix poinģons de ce décor subsistent mais leur datation demeure imprécise : qualre motifs gréométriques - le bouton pointé, le double cercle, l'arceau et le tortillon (fig. 10. E2. Ë). F1, L1) n'ont donné lieu à aucune comparaison et la chronologie des autres sujets est, le plus souvent, incertaine. Ainsi la biche est altestée dès LIBLR'T'Vs et se perpétue, les signatures le prouvent, durant la seconde moitié du ${ }_{1}{ }^{\mathrm{e}}$ siècle ${ }^{49}$. L'oiseau, en revanche, est malaisément identifiable : le nodele le plus proche paraît, sans doute, un poinçon de LIBBRTVS, repris par BVTRIO, puis

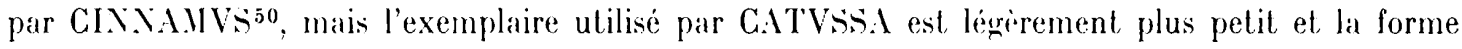
de ses paltes un peu différente. La feuille cordiforme, d'autre part. n'a été ailleurs identifiée par G. B. Rogers que dans un décor anépiographe atlribué à un fabricant de la seconde moitié du siècle. Enfin, les autres molifs secondaires, bien que mieux connus, n'offrent pas de grandes ressources pour la chronologie : le gros cordon perlé (fig. 10, A1), utilisé dejja par AVs'TRVsist, figure aussi au répertoire de potiers de la fin du siécle. En revanche, les références du cordon tireté (figr. 10, A:3) sont nombreuses, mais concernent souvent des modiles anépigraphes et dont la datation n'a pas encore été précisée: : il fut employé sûrement pendant le deuxième quart du siècle ${ }^{52}$ mais sa durée exacte reste indéterminée. L'ove (fig. 10, B1) apparait chez BVTRIO principalement, et sur des pièces signées d'un nom beaucoup moins célèbre : GRANIVS. Ainsi, l'ensemble des poinçons ratlache incontestablentent ce calalogue à la première moitié du ne siecle et le seul qui échappe à cette définition (la feuille) est rare et attesté, jusqu'à présent, par un exemplaire non sizné ; cependant la méconnaissance de la durée réelle de chaque motif interdit de supposor avec sérurilé une chronologie haute pour notre moule. De plus, si l'on considere le délail de l'ornementation, on voit, malgré les lacunes du décor, que les boucles coupées du rinceau sont totalement dépourvues des fantaisies qui

$48(0.1214==$ I). 675.

49 0. 1752A ? : 1). *79. Les listes d'oswald et de bechelette, completies par les planches de S.-S., attestent

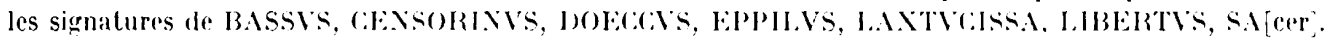

5) $0.2306=1) .101 \%$.

5l Fenille cordiforme : J74?; cordon : $\mathrm{A} 3$.

52 Cordon : A 15 ; ove : B 109 . 


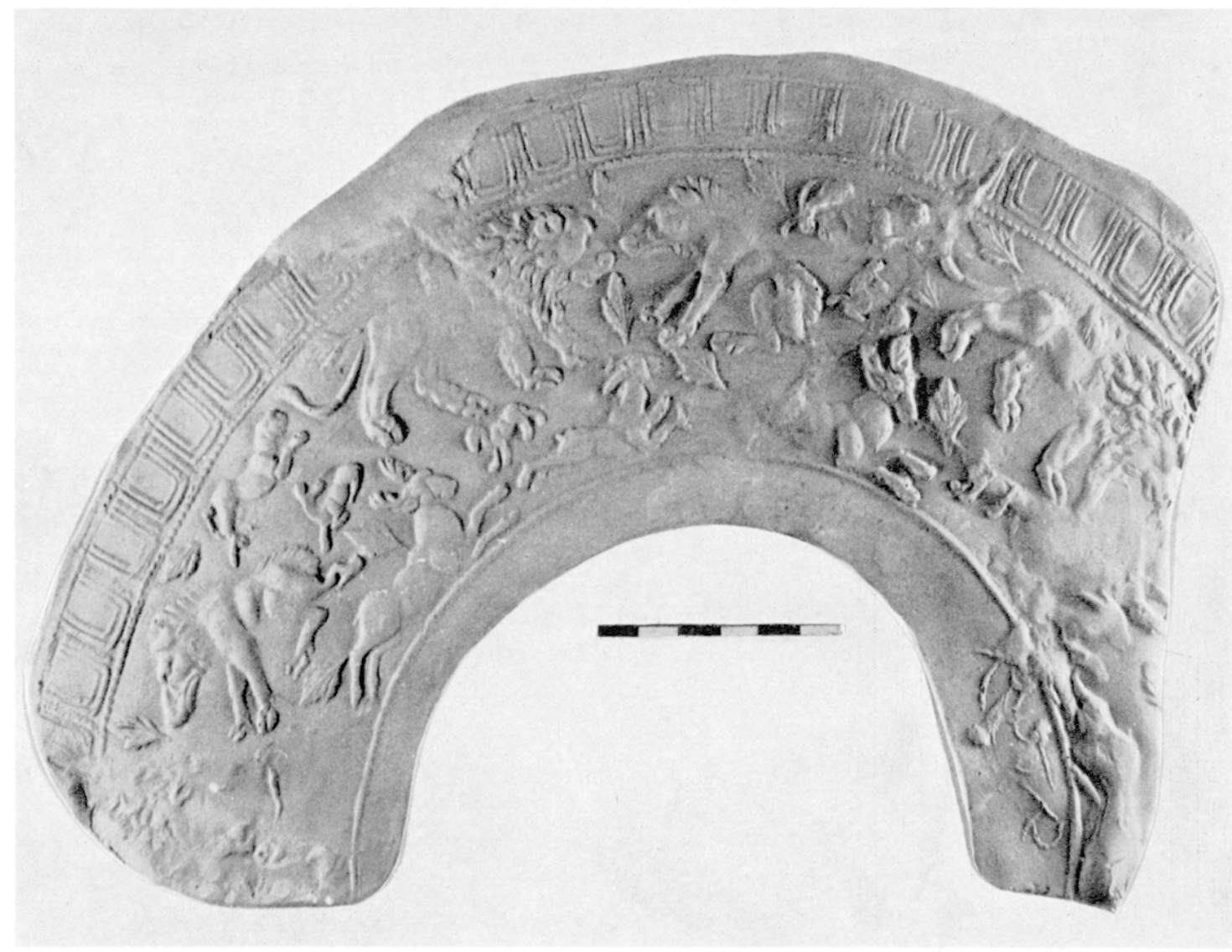

4 Voule de CATVSSA : empreinte de la moitio du bol 6599.3 et de la signature.

surchargent ce type de panneaux durant la première moitié du siède : imbrications, épaisses frises de séparation, semis végétaux. Én revanche, on pourra au moins noter la même sécheresse sur un vase signé par CINTVSIIVS, daté de la fin du siècle et comportant, également des boucles coupées ${ }^{53}$. Ces comparaisons, trop rares sans doute pour autoriser des conclusions entièrement assurées, permettent du moins de marquer une nette différence entre la technique de G.ITVSSit et celle d'un certain nombre d'artisans, que l'on situe sous les règnes de Trajan et d'Iladrien.

Les trois derniers moules (fig. 4 et 6 ) relèvent du mème genre : le style libre. Deux d'entre eux ont un répertoire comparable, constitué principalement par une série d'animaux, sauvages pour la plupart.

Selon une habitude commune aux potiers arvernes du ${ }_{i} \mathrm{e}$ siècle, le fond du décor est semé d'un petit ornement, que l'artisan utilise pour meubler les vides. Lin la circonstance, il s'agit d'une feuille pointue et découpée (fig. 10. H2 . que l'on rencontre déjà sur des vases ou moules portant la signature de BVTRIO, au moins dans des frises a métopes ${ }^{54}$. Wans la ventilation des poinçons, le bestiaire, comme il est naturel. l'emporte sur les ornements géométriques ou végétaux. Le grand moule comporte (figr. 1,$1 ; 4$ ), outre la feuille de BVTRI(1, neuf molifs : le cordon de grosses perles - visible déja sur la matrice au grand rinceau --- el un ove (B 165) propre jusqu'a présent à CATVSSA (fig. 10, B3). Les animaux (fig. 10, gl, g2, hl, il, j1, j2, l1) - un beau lion, une petite lionne, un

53 S.-S., pl. 164, 7. La signature est celle d'un finisseur, elle est attestee sur le bord de pieces dans le stỵle de CINNAMVS, ALBVCIVS, SERVVS III.

54 Feuille : 11151. 


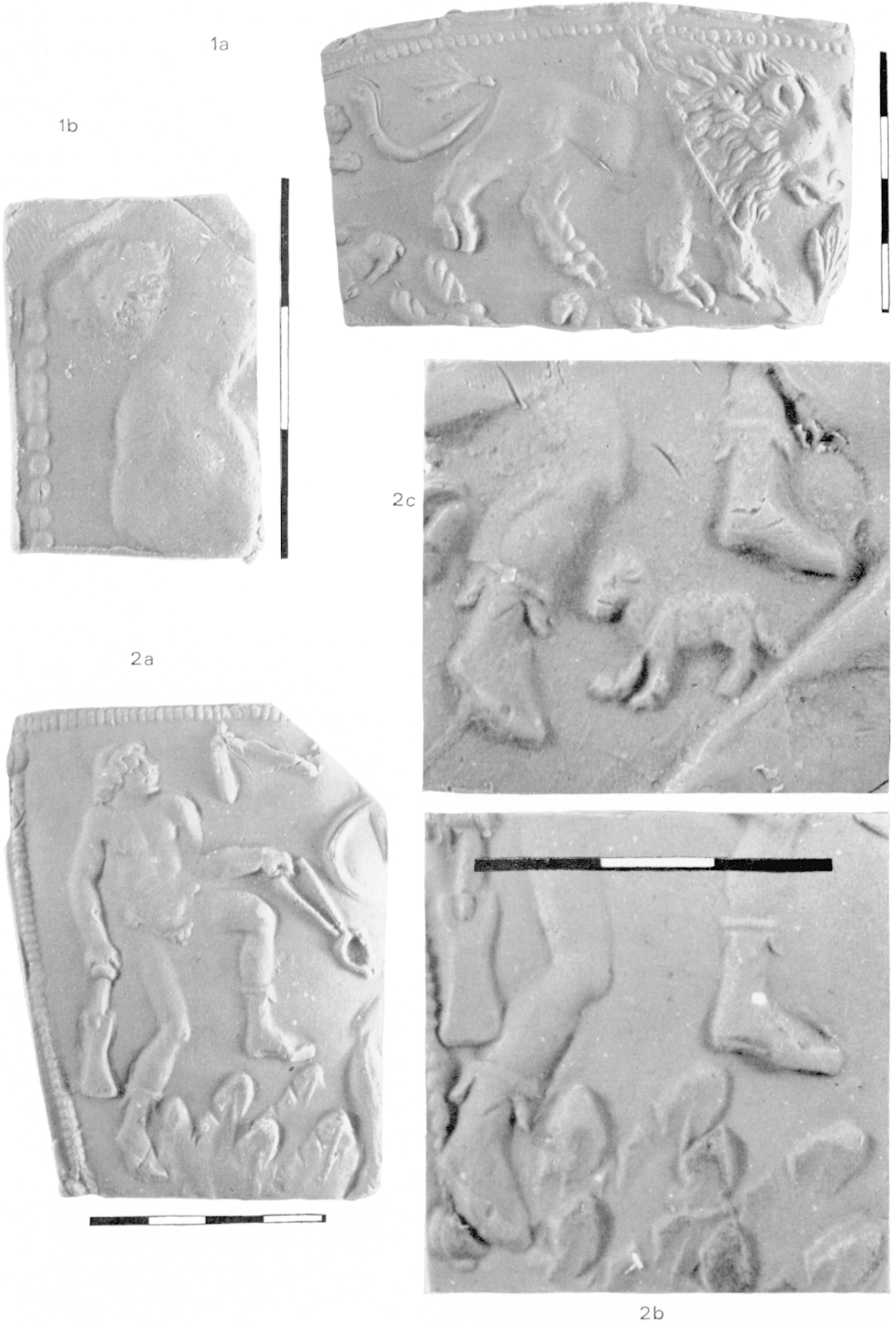

5) Poincons et ditails. 1 a: le lion, empreinte du moule 65993 (noter la surimpression qui allonge la patte postérieure gauche et l'excroissance sur l'échine); 1 b: agrandissement de li tête (?) plantée sur le dos de l'animal; $2 a$ : le chasseur travesti en Vulcain, empreinte du moule de CINXAHVS 32:257; $2 b$ : ag̣randissement des jambes d'après ce moule ; $2 c$ : le mêne detail daprès le moule trés usé de cATVSSA, P..l. 2. 
sanglier, un grand cerf bondissant, la biche au repos du rinceau, un petit bélier et une sorte de

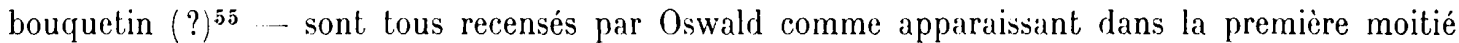
du siècle ${ }^{56}$, principalement dans l'atelier de LIBERTVS-BVTRIO (cerf, biche, bélier, sanglier), mais également dans celui de SACER (biche, sanglier ${ }^{57}$, lionne, bouquetin (?)). L'un des poinçons : le lion marchant à droile, paraîl encore peu connu à Lezoux, où Oswald ne l'a signalé que sur des vases anépigraphes, attribués à BVTRIO et BIRRANTVS. En l'absence de la moindre signature et de toute autre information, on peut hésiter au moins sur l'attribution à BVTRIO, compte tenu du nombre important des motifs - animaliers ou végétaux - communs à ce potier et à CATVSSA. La nature exacte du sujet représenté, en tout cas, n'est pas claire : la reproduction, inexacte, que donne Oswald, outre qu'elle modifie la forme particulière des pattes de l'animal, ne restitue pas un détail de la matrice. Le dessin, en effet, suggère que l'abondante crinière du faure se développe fort avant le long de l'échine. En fait, l'examen de notre moule (fig. 5,1 ) révèle une toison luxuriante mais arrêtée normalement au niveau de l'omoplate et une sorte de masse globuleuse saillant au milieu de l'épine dorsale. Cet appendice fait parlie du poinçon, car il reparait juste à la même place sur les trois exemplaires qui nous restent du lion et subsiste aussi sur la mauvaise reproduction du même modèle, que l'on retrouve à Rheinzabern, dans l'atelier de COBNERTVS III58. L'observation attentive de l'empreinte la plus nette permet de croire qu'il pourrait s'agir d'une tête de félin (lionne ou panthère), plantée verticalement sur le dos du lion (fig. 5, 16 ). Est-ce la trace d'un animal placé au second plan, sur un décor primilif, et dont la copie a fait disparaitre le reste? Si l'on considere le soin apporté au modelé de notre poinçon matrice, on imagine difficilement le maintien involontaire d'un détail parasite. L'hypothèse d'un lien organique entre cette tête et le corps du lion -transformé en une sorte de chimère - semble techniquement plus vraisemblable, mais je n'ai pu encore trouver de justification à celte représentation monstrueuse.

Quant à la durée de ce répertoire, nous noterons que trois des poinçons : le bouquetin (?), le sanglier et le lion, n'ont pas encore été attestés à Lezoux, pour la seconde moitié du II $^{\mathrm{e}}$ siècle. Toutefois, l'un des motifs, au moins, a pu connaître ailleurs une plus longue survie : le lion, en effet, ainsi que la lionne, le cerf et la biche, est repris à Rheinzabern par COBNERTVS III, potier daté par P. Karnitsch de la période $140-180^{59}$.

La disposition des motifs dans le champ se fait en deux zones principales : en bas du décor, le cerf et le bouquetin (?) alternés se poursuivent; au-dessus de cette frise, le lion marchant affronte le sanglier assis. Les petits poinçons (bélier, biche, lionne), sans rapport, par le registre ou les proportions, avec les grands sujets, et sans justification anecdotique apparente, se répartissent dans les blancs.

Du petit moule de la chasse (fig. 1,$2 ; 6,1$ ), peu de choses à retenir, sinon deux nouveaux motifs, qui s'ajoutent à quatre poinçons connus (lion, lionne, bélier, feuille) : un ours bondissant vers la droite (fig. 10, m2) et un chasseur (fig. 10, a $3^{\prime}$ ). La forme des pattes et l'étirement du corps apparentent le premier à un modèle reproduit dans Cientral Gaulish Polters, sous la signature de PATERNVS

55 Cet animal, classé parmi les caprins, sans doute à cause dn la longueur de son cou et de ce qu'on peut prendre parfois, à la rigueur, pour des cornes effilées et légèrement courbées vers l'arrière, ressemble, en fait (à la réserve du cou) par la morphologie générale, les proportions respectives des pattes avant el arrière, à un lièvre. J. Déchelette (op. cit., II, p. 144, no 968 j le classe parmi les quadrupèdes indéterminés.

56 Cerf : $0.1720=$ D. 852 ; biche : $0.1752 \mathrm{~A} ?=$ D. $879 ;$ bélier : $0.1868=$ D. 895 ; sanglier : $0.1664: \ldots$ D. $832=$ S.-S., pl. 84,17 et 90,2 ; lion : $0.1372=1$ J. 733 ; lionne : $0.1518=\mathrm{D} .799=$ S.-S., pl. $82,4,84,16,117$, 10, 153, 3, 156, 5 ; bouquelin ? : S.-S., pl. 82,1 et $3,84,17$ (différent de 0.1842 et 1843 ).

57 Ce dernier apparaît également sur un vase dans le style dit de IOENALIS (J. R. TrRrissF, Les céramiques sigillées gallo-romaines des Martres-de-Veyre (Puy-de-Dôme), XIXe supp. à Gallia, Paris, 1968, pl. Xl., 419).

58 op. cil., T 11 et T 11 a.

59 Certains d'entre cux, d'une nettete parfaite (la biche : T 110 ; la lionne : T :35; et le cerf : T 98), pourraient faire croire à une origine commune des poincons ; un autre, tres abâtardi, le lion, semble impliquer une copie de mauvaise qualité. En fait ces différences s'expliquent, aussi bien, par l'état accidentel du matériel de liheinzabern recensé, et le caractère variable et imprécis de l'échelle des reproductions publiées (cf. Ch. Fıschwr, op. cil., p. 5-6) ne permet pas de comparer la taille de ces motifs et celle de leurs analogues arvernes. 
et, probablement, d'ALBVCIVs, et aux types 1620 et 1621 d'Oswald, plus tôt altestés ${ }^{60}$. Cependant, l'animal est trop incomplètement conservé et ses pattes trop détériorées pour permettre une identification sùre du poinçon, alors que le sujet est très banal, à partir du deuxième quart du siècle, et que certaines représentations graphiques, demeurent insuffisamment précises. Le chasseur, lui, est bien connu, quoique rarement figuré, mais sa qualité exacte n'a pas été précisée : il s'agit, en effet, du personnage publié par Oswald sous le no 65 -... d'après une matrice anépigraphe et considéré comme un Vulcain (fig̣. $\overline{5}, 2 a)$. Le moule original, que j'ai eu entre les mains, représente un combat entre un guerrier cuirassé et un personnage nu portant des bottes de chasse ${ }^{61}$. Ce motif de chasseur a été complété, en la circonstance, par l'adjonction d'outils ou d'oljets imprimés à l'aide d'aulres poinçons : des pinces et un objet. énigmatique (gouvernail ou pelle à feu?), différent, en tout cas, de la représentation que donne l'Index. Outre la similitude générale des jambes (seules conservées) - mouvement, taille, proportions , nous avons, comme preuve de l'identité du personnage de CATVSSA et de celui de l'autre matrice, de petits détails communs aux deux moules: en particulier, un défaut qui affecte les quatre empreintes du pied droit subsistant sur nos deux tessons (fig. $5,2 b$ et $c$ ). Les deux potiers employaient donc le même matériel ou

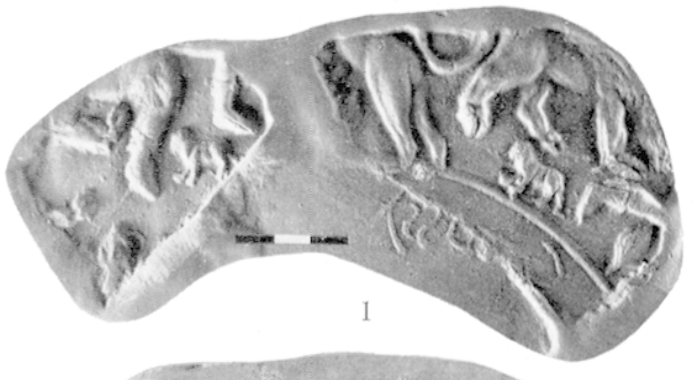
utilisaient les services d'un fabricant de poinçons poussant jusqu'à la perfection la production en série. Quoi qu'il en soit, l'ensemble du décor du fragment anonyme permet d'attribuer la pièce, sans hésitation, à CINNAIVS. Et il semble, d'ailleurs, que le mème sujet, dépourvu cette fois des accessoires qui en faisaient un Vulcain, ait déjà été publié avec la signature de ce potier : le personnage nu, botté, associé au mème guerrier cuirassé, parait sur un fragment de bol signé CINNAIII et provenant d'York ${ }^{62}$. Le mouvement du corps, en tous points identique, interdit de penser qu'il puisse s'agir d'un autre motif, mais ce dernier a été retouché : sur le mème cou trop court, enfoncé dans les épaules, le visage de l'hornme est tourné vers l'arrière. Le potier a-t-il lui-mème apporté cette modification, ou le relief, un peu flou, a-t-il abusé le dessinateur? : c'est ce que je ne saurait dire, faute d'avoir vu l'objet. Ainsi, GATVSSA et CINXIIIVS' ont utilisé l'un et l'autre ce modèle. Ils ne sont pas les seuls et n'étaient peut-ètre pas les premiers a le faire : un fragment de moule du Yusée de Saint-Germain porte le même décor ; il est anépigraphe, mais altribuable au potier $\mathrm{X}-5^{63}$.

Le dernier exemplaire de la série, de même style, mais d'inspiration toute différente, représente un groupe de dauphins et de tritons sur un fond semé de grands lleurons (fig. 1,4;6,2). Tous les motifs, à l'exception du cordon tireté qui souligne les oves, sont nouveaux dans le répertoire de

60 (if. S.-S., pl. 106, 22 et 123,$35 ; 0.1620$ : style de BVTRIO, (:IXX.MIS :Lezoux et signature de MEDETVS-RANTO (Blickweiler; ; O. 1621 : MEJETVS-RANTO Blickweiler Je n'ai pu encore vérifier que la grande ressemblance du motif avec celui qui figure sur un vase inédit de BVTRIO, au .lusée des Antiquités nationales.

61 O. 1.40. Le dessus du cràne lisse, alors que la chexelure bouclée auréole le visage, peut faire croire que le personnage porte une petite calotte ou que sa coiffure est retenue par une bandelette (cr. le dessin de J. Dícheistre, op. cit., II, no $42=.1 \mathrm{AN} 32257$;

62 S.-S., pl. 163, 68 .

63 Cette matrice inédite, identifiée déjà par G. B. Rogers, porte le grand losange propre à ce potier, ainsi qu'un cratère pansu souvent utilisé par lui. 
CATVSSA. L'ove, peu connu (fig. 10, B2), n'est, en dehors de cet atelier, attesté avec aucune signature sûrement identifiée,; le fleuron (fig. 10, G1) est, actuellement, signalé par G. B. Rogers comme exclusif. La faune marine, en revanche, paraitt un peu plus banale : un triton comparable (fig. 10, dl et dl"), sinon identique, est à la mode entre 150 et 190 ; deux des trois dauphins (fig. 10, ul et u2), au moins, apparliennenl au répertoire de BVTRIO puis sont utilisés jusqu'à la fin du siècle ${ }^{64}$. Le décor a des proportions modestes, étant données les dimensions du moule. Son caractère le plus notable, outre la remarquable fraîcheur du modelé, est sa relative rareté : si les animaux ou monstres marins sont fréquents dans les métopes, en revanche, dans les frises de style libre elles-mêmes beaucoup moins nombreuses que les compositions en rinceaux ou en panneaux - les thèmes marins représentent une faible minorité. Ainsi, parmi les exemples publiées par J. A. Stanfield et G. Simpson, on ne relève, au milieu des multiples chasses, que les produits de deux potiers utilisant le même type de répertoire dans le même genre de décor : IVSTVS et ALBVGIVS ${ }^{65}$. GASVRIVS et SERVVS III, peut-être SILVIO, mêlent des bateaux à ces frises; CENSORINVS suggère de légères métopes ${ }^{66}$. Cet invenlaire ne prétend évidemment pas être exhaustif, mais il peut au moins donner une première idée de la pauvreté relative du matériel de même espèce.

Nous voyons que la série des moules Plicque fournit un échantillonnage varié des possibilités d'un atclier que nous avons supposé unique sur la foi de signatures semblables. Il apparaît, à l'examen, que les rapports entre les différents décors sont très inégaux (cf. tableau, p. 95) : les deux vases aux chasses ont un répertoire commun pour une grande part et le plus complet a, par ailleurs, des relations avec le moule au rinceau (cordon perlé et biche). Ces trois formes portent, en outre, la même signature, tracée de la même écriture courante. Pour le reste du groupe, les liens sont assez ténus : à l'intérieur de la série géométrique, on trouve le même arc dans les deux rinceaux (lagène et bol 37), la même rosette dans les deux frises à métopes (lagène et fragment de 37), peut-être le même gros cordon de perle sur le moule au rinceau et le fragment aux panneaux, mais aucun poinçon figuré ne paraît sur plus d'une matrice. Le décor aux dauphins ne s'apparente qu'au rinceau par le cordon tireté qui souligne les oves. L'inventaire fait donc ressortir d'abord l'hétérogénéité de plusicurs de ces pièces : sans l'analogie de leurs signatures, elles n'auraient aucun rapport très apparent; d'autre part, le bol au rinceau est le seul, ou peu s'en faut, à comporter un répertoire mixte et à établir un lien entre les frises libres et les compositions géométriques. On ne saurait, toutefois, tirer argument de ces dissemblances pour écarter dès maintenant du groupe l'un ou l'autre des moules, même le plus original par son décor et sa signature : le bol aux dauphins. En effet, la spécificité de certains poinçons figurés n'a pas lieu de surprendre : les vases à thèmes - décors de chasse ou décors marins excluent normalement, par leur inspiration même, la plupart des personnages que l'on voit dans les métopes; et, si les frises géométriques sont, en revanche, accueillantes aux sujets les plus divers, nous sommes limités, en la circonstance présente, par la qualité de l'échantillonnage : les matrices diffèrent par leur forme - le caractère exceptionnel de la lagène peut, a priori, influencer l'ornementation de l'objet --, et par la conception du

64 Ove : B 136 ; fleuron : G 72 ; triton : cf. $0.21=$ D. 17 =S.-S., pl. 101 , 4, etc.; grand dauphin (d.) : O. $2384=$ S.-S., pl. $77,2,89,9,111,13$ et 17 ; grand dauphin (g.) : $0.2393=$ D. 1052 ; petit dauphin : cf. 0.2392 et D. 1051 ?

65 S.-S. : ALBVCIVS : pl. 121, 8; IVSTVS : pl. 111, 12-14.

66 Ibid. : CASVRIVS : pl. 137, 62; SERVVS III : pl. 138, 2 ; SILVIO : pl. 77, 3; CENSORINVS : pl. $101,4$. 


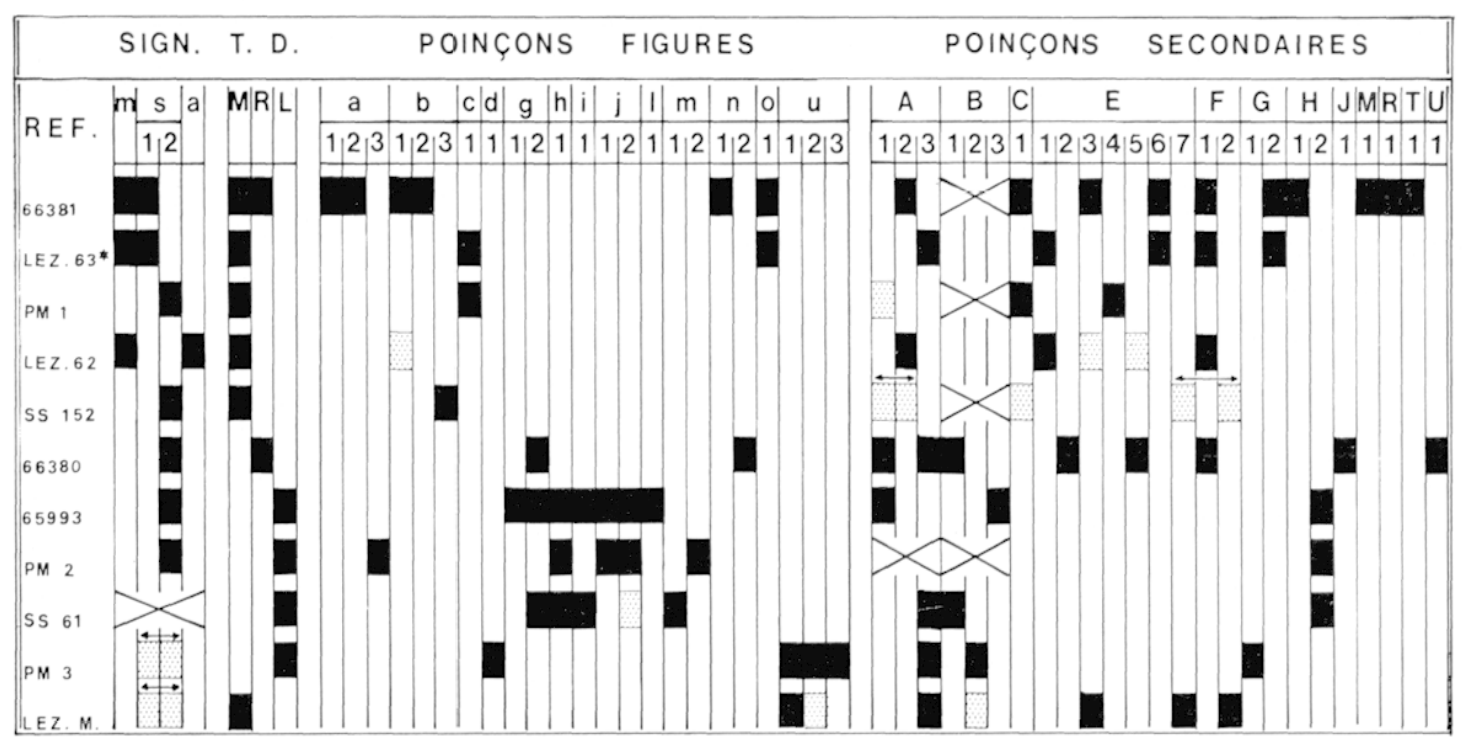

Sigles et conventions graphiques :

Signalures

$\mathrm{m}:$ manu
$\mathrm{s} 1:$ Calusa
$\mathrm{s} 2:$ Calussa
$\mathrm{a}:$ Caluassa

Type de décor (T.D.)

$\mathrm{M}$ : métopes

$\mathrm{R}$ : rinceau

L : «libre"

Table de concordance * :

$\begin{array}{ccc} & 0 . & D . \\ \text { a1 } & 153 & 100 \\ \text { a2 } & 1057 & 616 \\ \text { a3 } & (65) & 42 \\ \text { b1 } & 277 & 172 \\ \text { b2 } & 1197 \mathrm{~A} & \\ \text { b3 } & 812 & 475 \\ \text { c1 } & (1214) & 675 \\ \text { d1 } & 21 & (17) \\ \text { g1 } & 1720 & 852 \\ \text { g2 } & (1752 \Lambda) & 879 \\ \text { h1 } & 1868 & 895 \\ \text { i1 } & 1664 & 832 \\ \text { j1 } & 1372 & 733 \\ \text { j2 } & 1518 & 799 \\ \text { I1 } & & \\ \text { mI } & 1574 & \\ \text { n1 } & 2234 & 1041 \\ \text { lo1 } & 2344 \mathrm{~A} & \\ \text { uI } & 2384 & \\ \text { u2 } & 2393 & 1052\end{array}$

\begin{tabular}{|c|c|c|}
\hline S.-S. & & $R$. \\
\hline & $A 1$ & A 3 \\
\hline & $\Lambda 2$ & $\Lambda 13$ \\
\hline & $\mathrm{A} 3$ & A 15 \\
\hline & $B 1$ & 13109 \\
\hline & $\mathrm{B} 2$ & 13137 \\
\hline & B33 & B165 \\
\hline & $\mathrm{Cl}$ & C214 \\
\hline & Gl & G. 72 \\
\hline & (32 & G201 \\
\hline & III & H 78 \\
\hline & H2 & H151 \\
\hline & $\mathrm{J} l$ & $\left(\begin{array}{ll}\mathrm{J} & 74\end{array}\right)$ \\
\hline & MII & if 38 \\
\hline & RI & I 65 \\
\hline 82,84 & $\mathrm{~T} 1$ & T 5 \\
\hline
\end{tabular}

A 3

$\Lambda 13$

3137

3165

C. 72

G201

( $\mathrm{J} 74)$

If 65

T' 5

- Les trois moules de Lezoux (L.ez. 62, L.ez. 63, Lez. M.) ne sont utilisés ici que dans la mesure où ils témoignent de l'emploi de poinçons par ailleurs connus dans le répertoire de CATVSSA.

- Identifications plus ou moins douteuses indiquées par des parenthèses. 
décor (rinceaux et métopes) ; la taille de l'un des bols (fig. 1,3) interdit les figures de grandes dimensions; ; enfin, l'un des fragments est si petit qu'il réduit considérablement le champs des comparaisons. I'autre part, le nombre des exemplaires n'est que de trois. L'amabilité de B. Hartley m'a permis d'étendre mon information et d'établir indirectement de nouveaux liens à l'intéricur de la série dont je disposais. Le relevé des décors de trois moules à métopes provenant de Lezoux ${ }^{67}$, et dont aucun n'a fait l'objet d'une publication détaillée ${ }^{68}$, élargit, comme on pourait s'y attendre, le catalogue des poinçons de CATVSSA et, surtout, révèle d'intéressantes parentés entre ce groupe, relevant du style à métopes, et plusieurs des matrices Plicque (cf. tableau, références Lez. 62, Lez. 63, Lez. M.). Ainsi, le cordon, l'arceau et, au moins, le motif de la Vénus ${ }^{69}$ sont communs à la lagène et à un moule 37, signé maladroitement CATVASSA $M^{70}$ et orné, semble-t-il aussi, du double cercle du vase au rinceau. Lne autre petite forme 37, portant l'inscription [c]ATVSA MA présente, à la fois, le cordon tireté, une partie du décor de la lagène - arceau, coq dans son cercle double, feuille composée - et le masque figuré sur notre petit fragment à métopes. Grâce à ces deux nouveaux échantillons, s'établit donc une relation à l'intérieur d'une série géométrique (cf. tableau, lignes 1-4) comportant les signatures cursives : CATVSAS MANV, [ . . ]ATVSA MA, CATVASSA MA, [ . .]AṬVSSA (fig. 11, I, i, f, e, c), et englobant la lagrène ot des bols 37. Le troisième exemple s'apparente presque exclusivement à la frise marine, dont il reprend en partie le répertoire ${ }^{71}$ : on retrouve en effet le grand dauphin à droite et, sans doute (il ne reste que la queue), le grand dauphin à gauche ; de plus le cordon tireté souligne un ove comparable à celui de la matrice de style libre, mais, peut-être, légèrement plus haut; enfin, le début, seul conservé, de la signature : CATV [...] rappelle d'assez pres le graffite incomplet sous les dauphins (fig. 11, I, h et g) : $C$ court, $A$ raide, $T$ à harre modeste, $V$ amorcé par une haste oblique. Le reste des motifs, neuf en tout, paraît constitué presque exclusivement de poinçons étrangers aux moules précédemment rités ${ }^{72}$. Ainsi, parmi les décors portant une signature attribuable à GATVSSA, croit-on voir se dessiner, avec ces deux pièces, l'amorce d'un sous-groupe sans rapport autre que lâche (cf. tableau, lignes 10-11) avec l'essentiel de la série ici inventoriée.

Tel qu'il se présente actuellement, après l'examen des moules Plicque, le répertoire de cet atclier comporte quarante-et-un poinçons sûrement identifiés ${ }^{73}$. Les trois dessins

67 L'un se trouve au musée, les autres proviennent des fouilles de 1962 et 1963 ; ils sont signés respectivement CATVI...], CATVASSA MA et [.]ATVSA MA (avant cuisson?).

68 La photographie de l'un d'eux est toutefois connue (supra, n. 4).

69 La qualite du releví ne m'a cependant pas permis de m'assurer formellement que cet exemplaire n'etait pas légèrement plus haut, et plus laroge, fur notre poincon. Aux figures déjà identifices on doit ajouter un Apollon proche de (). $73 . \mathrm{A}$ of un masfue de Pan cher a I.IBEEIYTVS et BVTRIO (O. 1215). Les motifs secondaires comprennent, entre autres, deux fleurons (dont (;107, un nerud, un petit cercle, un ove (B 135) non encore signalés dans ce répertoire.

70 B. Hartley n'est pas certain que cette signature ne soil pas gravée après cuisson. Si cette opinion se trouvait solidement confirme plutot que l'hypothese d'une gravure sur terre tres siche, il faudrait expliquer la similitude du répertoire des moules de L.zoux marques CATVASSA MA et [.]ATVS. MA et de celui de nos moules 66381 et P.M. 1.

71 Ce dernier comporte, outre des panneaux coupés, des métopes contenant de grands médaillons à personnages.

72 Cordon : $\triangle 15$; ove:B 136 ?; disque : $:$ : 304 ; homme debout : cf. $0.529 / 530$; femme appnyée a une colorne : O323; homme nu : (). 539 ; cheval marin : (). 52.

73 Certains : le grand ove carre, la roselte à dix pétales, le noud de la lagène, le fleuron, n'ont pas, pour le moment, d'autre attestation connue. 
provenant de Lezoux permettent d'ajouter au catalogue -..- non sans réscrves pour les motifs secondaires que je n'ai pu vérifier - une vingtaine de nouveaux sujets ${ }^{74}$. Dans la mesure où ces derniers ont été identifiés, les limites connues de leur emploi ne diffèrent pas sensiblement de la durée des modèles étudiés ici en détail, mais il est souhaitable qu'une critique soigneuse des objets eux-mêmes et de leur décor permette une évaluation exacte des ressources nouvelles qu'offre ce matériel, récemment découvert. D'autre part, l'inventaire provisoire ne serait pas complet, si je ne signalais deux vases, déjà publiés, appartenant à la mème production et fournissant deux motifs fiğurés. L'un (cf. tableau, S.S. 152), signé ${ }^{75}$, révèle l'emploi, dans une composition à métopes, d'une Fortune (fig. 10, b3), apparemment utilisée aussi par PATERNVS. L'autre, réduit à deux tessons anépigraphes, dont le Dr. G. Simpson a eu l'amabilité de me communiquer les frottis, était classé parmi les produits imputables a BVTRIO ${ }^{76}$. Toutefois les fragments, qui portent, sous l'ove du moule au rinceau et le cordon tireté, les vestiges d'une chasse, ont conservé la biche, le bélier, le sanglier et peut-ètre la lionne de CATVSSA (ou sa reproduction réduite) sur un fond semé de la feuille découpée commune à ce potier et à BVTRIO. La nouvelle attribution me paraît très probable (cf. tableau, lignes 7-9), maintenant que le répertoire de notre potier est mieux connu et Miss Simpson partage cette opinion. Dès lors s'ajoute à une riche série d'animaux sauvages un ours marchant vers la droite (fig. 10, m1) : il faut, selon toute apparence, le rapprocher d'un poinçon, publié par Oswald, très caractéristique du fait de l'espèce de crinière qui couv re la nuque et les épaules de l'animal, et attribué aux styles de LIBERTVS et de IVLLINVS? ${ }^{7}$. Nous voyons, par ce premier aperçu nécessairement incomplet, que le répertoire de CATVSSA, sans briller par une excessive originalité, promet d'être assez riche. I'autre part, malgré une production peu abondante, ou fortuitement trop méconnue, ce potier manifeste, en particulier à travers les échantillons de la collection Plicque, une variété dans la mise en œuvre qui fait de lui, de ce point de vue, l'égal de producteurs plus largement réputés. Enfin, la qualité de certains des poinçons, surtout dans les frises de style libre, suggère des rapports plus étroits qu'il n'était prévu avec des ateliers de la première moitié du $\mathrm{II}^{\mathrm{e}}$ siècle. Pouvons-nous, par l'analyse de ses techniques de composition, définir plus complètement ses relations avec la manière de procéder d'autres décorateurs?

L'organisation générale des différents types de décors représentés sur les six moules, qui nous intéressent ici principalement, donne, au premier abord, et sans doute, en partie, à cause de la variété des supports et des motifs figurés, le sentiment d'une assez grande diversité. En fait les règles de composition sont simples et plus semblables entre elles qu'il n'y parait.

7.1 Ils se répartissent ainsi : deux oves, un noud, deux fleurons, un gros pois, trois petits cercles simples, un grand cercle double, un arceau double, une grande rosace, une feuille, un cheval marin, quatre personnages.

75 S.-S., pl. 152, 1, trouvé à Corbridge. La Fortune est signalée par Oswald (812) et 1)échelette (175). Malgré les patientes recherches aimablement effectuées par. J. P. Cillam, il n'a pas été possible de retrouver le tesson et de confirmer l'identite, probable, des details secondaires du decor (rosette, cordon perlé) el de certains des poincons dejà recensès.

76 Op. cil., pl. 61, 684. La cassure rendait difficile la determination exacte de la silhouette de la lionne d'apres le frottis.

77 0. 1574 . 
Les décors géométriques se fondent sur un partage régulier de la surface, par le moyen d'ares de cercles ou de cordons rectilignes : chacun de ces types de division figure seul sur un moule; ils sont combinés ensemble sur une troisième matrice. Le bol au rinceau (fig. 8,2) est décoré selon un rythme élémentaire, aisé à construire au compas : les parties conservées permettent en effet de reconstituer, sans doute possible, la succession de six doubles-boucles.

Cinq des courbes ouvertes vers le haut contiennent les restes d'une ornementation végétale : il en allait done sûrement de même de la sixième. Les fragments de deux feuilles semblables conduisent à supposer l'utilisation, au moins une fois sur deux, d'un mème modèle en forme de cocur. Pour des raisons analogues, il est logique d'imaginer une biche couchée dans chacune des boucles ouvertes vers le bas et une division de ces dernières en deux registres. Ce partage était marqué, nous le voyons, au moins une fois sur deux, par un cordon horizontal et la plaque supérieure occupée, selon le même rythme, par un oiseau inscrit dans un cercle double. Les éléments conservés sont assez modestes et ne fournissent pas de raisons de croire à autre chose qu'à la répétition monotone des mèmes motifs à six reprises. Toulefois les hasards des cassures n'excluent pas la possibilité d'une alternance de poinçons dans le cas de la feuille et celui de l'oiseau inscrit. Enfin, certaines parties du rinceau sont trop lacunaires pour permettre la moindre hypothèse.

La comparaison de l'économie d'ensemble du décor avec la composition d'un certain nombre de frises du même genre amene d'abord à constater que de nombreux décorateurs utilisaient le grand rinceau tantòt romme une surcession de panneaux alternativement pleins et coupés, tantòt comme une série de simples métopes pleines, et meublaient ces espaces parfois de motifs ou personnages variés, parfois d'une alternance de sujets figurés et de végétaux ou de feuilles, parfois, simplement, de feuilles. Cependant, à l'intérieur de cette famille toutes les rariétés dans les combinaisons ne semblent pas avoir eu nécessairement le mème succès à la mème époque : ainsi, la boucle coupée tend à disparaître dans la seconde moitió du $\mathrm{II}^{\mathrm{e}}$ siècle, où on lui préfère le panneau plein, enfermant un médaillon inserit ${ }^{78}$, et à la mème époque les feuilles paraissent beaucoup plus fréquemment dans les panneaux que les motifs végétaux (vrilles, boutons, rosettes...) souvent utilisés au début du siècle ${ }^{79}$; en revanche, le médaillon inscrit parait rare à haute époque ${ }^{80}$. Il résulte donc de ces observations que le moule de CATVSSA se rattache par sa conception, à une tradition représentée surtout par des décors datés de la première moitie du siècle. On ne saurait néanmoins tirer des conséquences rigoureuses de cette seule remarque, dans la mesure où les poinçons sont incomplètement datés, où la sobriété du décor se rapproche

78 Les exemples fournis dans S.-S. et la publication de P. Karnitsch 'Ovilana, Linz, 1959, p. 142-238; prouvent

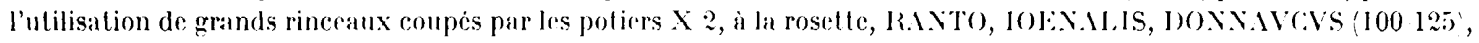

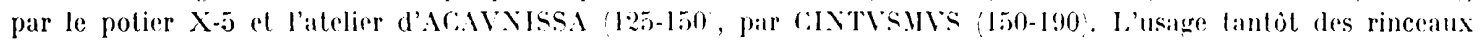
coupés, tantôt des rinceaux pleins apparaît dauns le style dit de MlEDETVS-MANTo (100-120), dans latelirr de

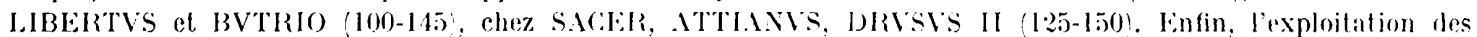

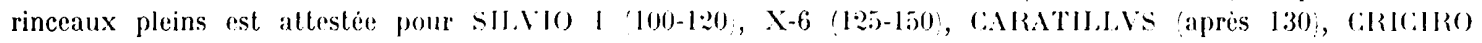
CINNAIVS (140-180), IVSTVS (150-180), PATERNS, AVENTIVS, LAXTVG:ISSA (150-190), bOECCVS, SERVVS II, CETTVS, BANVVS 160-195: X-6, P.ITERXVS, CINAMVS, IVSTVS, BANVV emploient volont iers de grands médaillons inscrits dans les courbes.

79 C'est, par exemple, ce qui distingur lr rinceau non coupé de SIIVIO) I de ceux de potiers de la fin du siecle (cf. S.-S., pl. 33).

80 In decor d'Acillisst s.s., pl. 81,31 montre une allernance de boucles coupres et de boucles a médaillon. Je n'ai, pour le moment rien tromié d'exactement comparable à la frise de cATVSSA. 


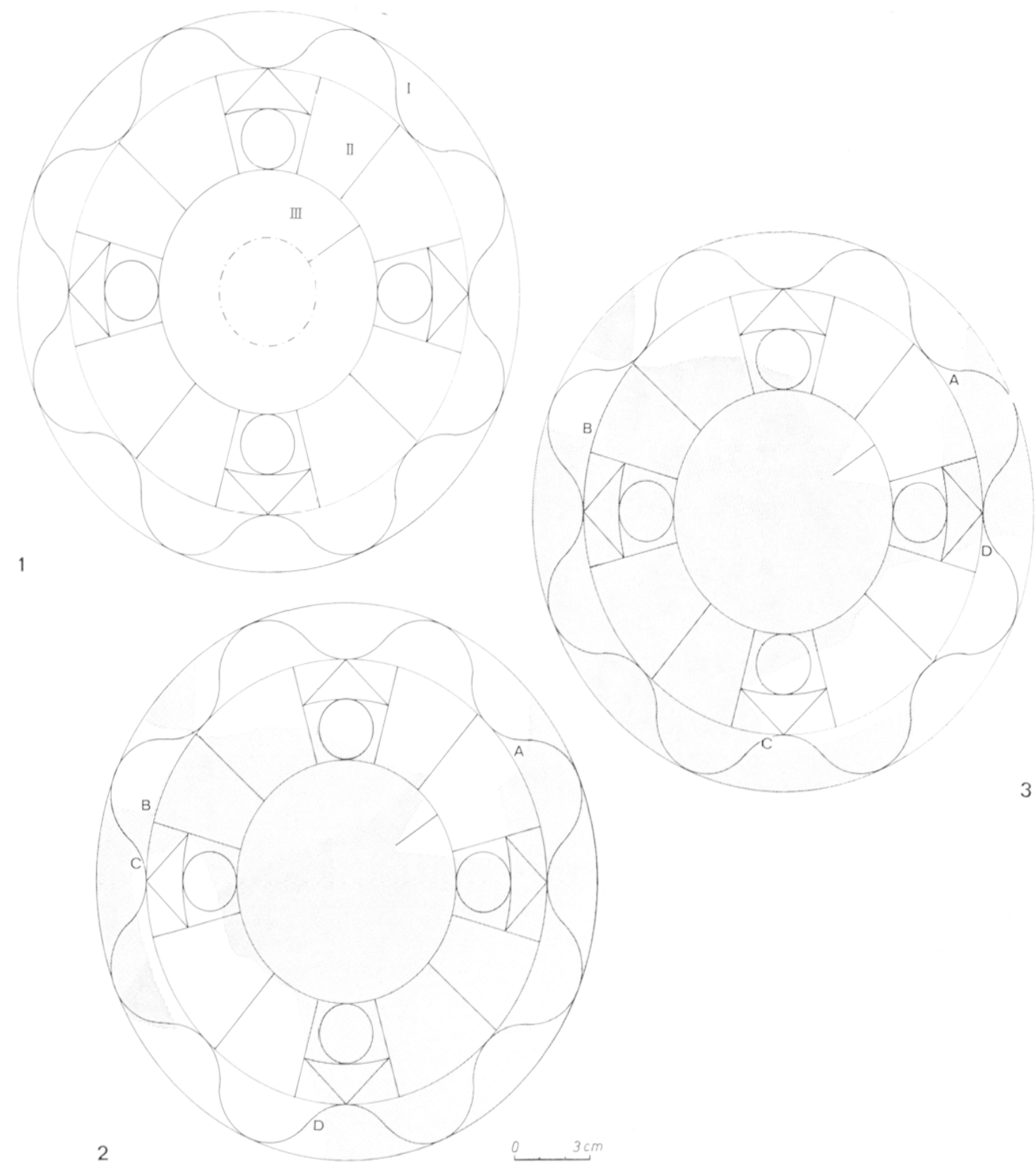

7 L.a lagène : schéma géométrique du décor. 1 : restitution des trois zones (en pointillé : limite hypothétique de III); 2-3: essais de localisation des fragments conserves ( 2 : supposition la moins vraisemblable; $3:$ hypothese la plus solide).

plutôt du style de potiers situés sous le règne d'Itadrien, voire plus tard, où, enfin, l'effacement des rinceaux coupés ne paraît pas complet chez les décorateurs les plus tardifs. Sans doute avons nous affaire à un style mixte ou de transition, mais une datation haute n'est pas actucllement plus fondée, compte tenu du nombre encore restreint des termes de comparaison, que l'éventualité d'une survivance plus tardive.

Le moule de lagène est le seul dont l'état de conservation autorise à reconstituer la composition du décor à métopes. La comparaison des parties conservées et de la circonfé- 
rence totale permet de retrouver la structure de deux registres (fig. 7,1$)$ : celui des grands panneaux et le rinceau.

Le premier est divisé en quatre parties et les deux ensembles de fragments subsistants ne contiennent rien qui conduise à imaginer une construction symétrique. En revanche, la succession des métopes, plus ou moins complètes, révèle l'existence d'une séquence de trois panneaux qui couvre un quart de la surface de la zone décorée et se répète dans le même sens. Nous disposons des restes d'un groupe de quatre et d'un groupe de cinq métopes consécutives, et la régularité des enchaînements ${ }^{81}$, l'espace libre entre la fin d'un ensemble et le début de l'autre, tout invite à rétablir les trois ou quatre panneaux absents, en respectant la même cadence. La restitution géométrique du schéma de cette bande se présente donc sous la forme proposée ci-contre (fig. $7,1, I I$ ) et la mise en place des fragments conservés peut être envisagée de deux façons (fig. 7, 2-3). Or les deux séries de métopes n'ont aucun point de contact et la structure graphique du décor n'impose pas une solution plutôt que l'autre, mais la forme du moule, en l'occurrence, guide le choix : l'épaississement du bord est de hauteur variable et passe insensiblement de son point le plus bas, en $\mathrm{C}(39 \mathrm{~mm})$ à $40 \mathrm{~mm}$ en $\mathrm{D}$, puis de $41 \mathrm{~mm}$, en A, à 43, en B. Seule la disposition envisagée en second lieu (fig. 7, 3) ménage entre $\mathrm{B}$ et $\mathrm{C}$ un intervalle suffisant pour permettre un passage très progressif (done compatible avec la pente générale) du point le plus haut : $\mathrm{B}$, au plus bas : C. Les restes des motifs décoratifs ne fournissent, pour cette zone, que des sujets identiquement répétés d'une séquence à l'autre. Toutefois, seuls deux exemplaires de la métope recoupée ont été suffisamment préservés pour qu'on sache qu'ils étaient identiquement occupés. Dans la disposition des fragments à laquelle je me suis arrêtée, les deux panneaux se trouvent face à face : on ne peut dont négliger l'hypothèse que, dans les deux autres cas, le même découpage contienne des éléments décoratifs différents, alternant avec les premiers. En revanche, les deux autres séries de métopes ne laissent pas place à une telle éventualité : les décors de même origine et qui sont construits sur cette quadruple répétition de la même suite de panneaux n'admettent, en règle générale, comme rythme d'alternance à l'intérieur de cadres identiques, que la succession de deux poinçons ${ }^{82}$. Or les fragments de la valve conservent, pour les métopes non coupées, des séquences consécutives décorées des mêmes sujets : l'hypothèse d'une alternance et, par conséquent, d'une quelconque diversification des motifs parait donc exclue.

Cette organisation, à l'encontre des recherches dont témoignent les plus beaux modèles des ateliers du Sud, est d'une extrême banalité : il n'est, pour en juger, que de la comparer à un certain nombre de décors sur bols 37, produits à Lezoux, à partir du règne d'Hadrien. Sans doute le nombre des panneaux par séquence varie-t-il, ainsi que le découpage interne de ces derniers, mais le mode de répétition et le rythme quaternaire sont identiques. Le petit fragment de moule 37 à métopes (fig. 8, 1), qui appartient à notre série ${ }^{83}$ est trop exigu pour que nous puissions juger de la structure du vase, mais les relevés communiqués par $B$. Hartley, prouvent que la répétition de séquences analogues ou absolument identiques était, pour CATVSSA, un procédé habituel ${ }^{84}$. Et les proportions des fragments reproduits par projection tronconique permettent de supposer avec la plus grande vraisemblance une division en quatre de la zone ornée. En allait-il de même

81 Il faut noter de petites irrégularités dans les dimensions des panneaux appartenant au même type. Ce défaut, plus ou moins apparent sur la plupart des vases à métopes, est dù pour une grande part, dans le cas présent, à l'inclusion de la signature.

82 C. S.-S., pl. 120, 1 ; C. BE்noxt, La composilion des décors géométriques à plan centré: contribulion à l'élude du style des vases sigillés à métopes, dans Rei cretariae romanae fautorum, Acta XIV, figr. 1 (en cours de publication).

83 P.M. 1.

84 C'est le cas, au moins, des matrices au cơ et aux dauphins précédemment analysées. 


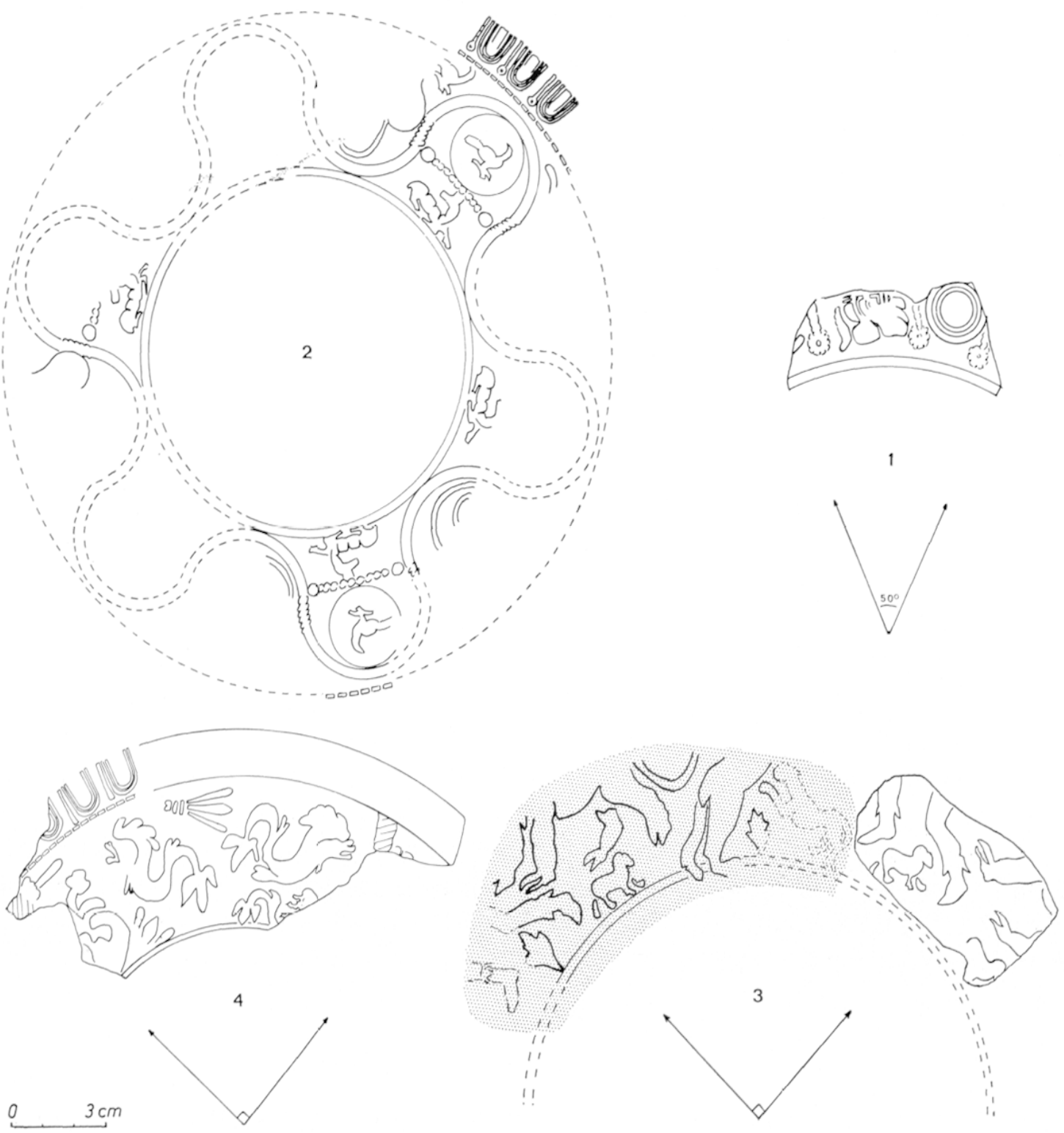

8 Schémas de décors. 1 : projection circulaire du fragment P.II. 1 ; 2 : restitution du rinceau du bol $66380 ; 3:$ projection circulaire du fragment P.M. 2 (en grisé et traits épais : séquence correspondant à un quart du décorj; 4 : projection circulaire du fragment P.I. 3. (Décors non inversés).

pour le registre supérieur de la lagène? Il ne subsiste que deux panneaux, et encore incomplets : on ne peut donc préjuger de la fantaisie ou de la monotonie de la composition à ce niveau.

Quant au rinceau, il semble que l'intention du potier ait été moins d'introduire une nouveauté que de soutenir discrètement le mouvement général des groupes du registre principal. La répétition régulière de la mềme double-boucle n'a rien que de normal et les fragments préservés de la destruction prouvent, en outre, la reprise du même oiseau et de la même grappe dans la même boucle, ainsi que celle de la rosette et du cercle (avec une irrégularité cependantj, dans la courbe complémentairc. Par ailleurs, la disposition 
des restes du rinceau par rapport aux métopes conservées et la comparaison du développement moyen d'une double-boucle et de la circonférence du moule autorisent à rétablir le déroulement complet de la frise : elle apparaît comme constituée de huit éléments semblables, deux sous chaque séquence de panneaux (fig. 7, 1,II-III). Je laisse à chacun le soin de décider si la combinaison rythmique des deux zones ornées révèle un certain souci d'harmonic ou une regrettable pauvreté d'invention. L'effet n'est pas déplaisant, mais notre ignorance quant aux problèmes techniques posés au potier par la construction du décor, et aux procédés et instruments par lui utilisés pour les résoudre, est totale : aussi ne savons-nous pas si le synchronisme rythmique est un effort qu'il s'impose ou une facilité qu'il se donne. Seule, peut-être, une enquête comparative assez large sur des compositions à registres superposés, au ${ }^{\mathrm{er}}$ et au $\mathrm{II}^{\mathrm{e}}$ siècles, dans le Sud et le Centre, pourrait nous faire pressentir la signification de probables différences.

Les trois derniers décors ressortissent au style libre. Ce mode d'organisation, qui apparaît à Lezoux, principalement, au II $^{\mathrm{e}}$ siècle, se définit surtout en opposition par rapport aux métopes. Alors que ces dernières se présentent d'abord comme une construction volontairement et visiblement géométrique de la surface, le style dit libre bannit les cordons, arcs et médaillons de toutes sortes. D'autre part le cloisonnement des panneaux attire l'attention sur l'équilibre décoratif du tout et implique un morcellement du sujet, même si l'ensemble, ou certaines parties de la frise ont, par ailleurs, une signification anecdotique, une inspiration commune. Au contraire, le déroulement continu de la zone ornée suppose d'abord une vision globale des motifs et explique probablement que les vases de style libre aient bien souvent un thème unique, immédiatement apparent : courses d'animaux, chasses, jeux du cirque, cortèges marins. En fait ce genre a, jusqu'à présent, été surtout caractérisé de façon sommaire et négative et englobe, si l'on considère, par exemple, le recueil, si riche, de J. A. Stanfield et G. Simpson, des séries de compositions fort différentes les unes des autres. Il n'entre pas dans mon propos de traiter ici une question complexe et qui nécessite nombre d'études de détail, mais le groupe de fragments que j'ai rassemblé permet déjà de définir l'une des formes de ces organisations non géométriques.

Le grand moule peuplé d'une foule d'animaux, assis ou en mouvement, a conservé un décor complet : malgré les lacunes qui affectent une moitié de la frise, il n'est pas un animal dont il ne reste, au moins, une petite trace. Aussi est-il possible, d'après la partie la meilleure et les vestiges subsistant sur l'autre, de restituer l'ensemble de la composition. Or, une fois passée l'impression première, au spectacle de ces corps enchevêtrés, on s'aperçoit vite que l'apparente confusion n'est que le masque d'une composition ordonnée ${ }^{85}$. La reproduction graphique schématisant les silhouettes, que je présente ci-contre (fig. 9), est une projection circulaire du décor : à partir de frottis, chaque élément a été mis exactement à sa place, par rapport au cercle limitant la base de la zone ornée. Il suffit de regarder le dessin pour voir que l'ensemble de l'ornementation se divise en quatre parties identiques,

85 Sur le caractère apparent de la liberté des décors on notera une remarque faite en passant par J. Déchelette, loc cil. : "Les vases de cetle catégorie offrent la plus grande ressemblance avec les vases à métopes présentant des figures séparatives, mais les divisions ne sont pas indiquées ou se distinguent à peine. "Voir également le style "sernilibre "de CIBISVS, distingué par W. Lutz (I)écouvertes à Ehl (Bas-Rhin) d'un lot important de sigillée de CIBISVS, dans Cahiers alsaciens d'archéologie d'art el d'histoire, XV, 1971, p. 65;,. 


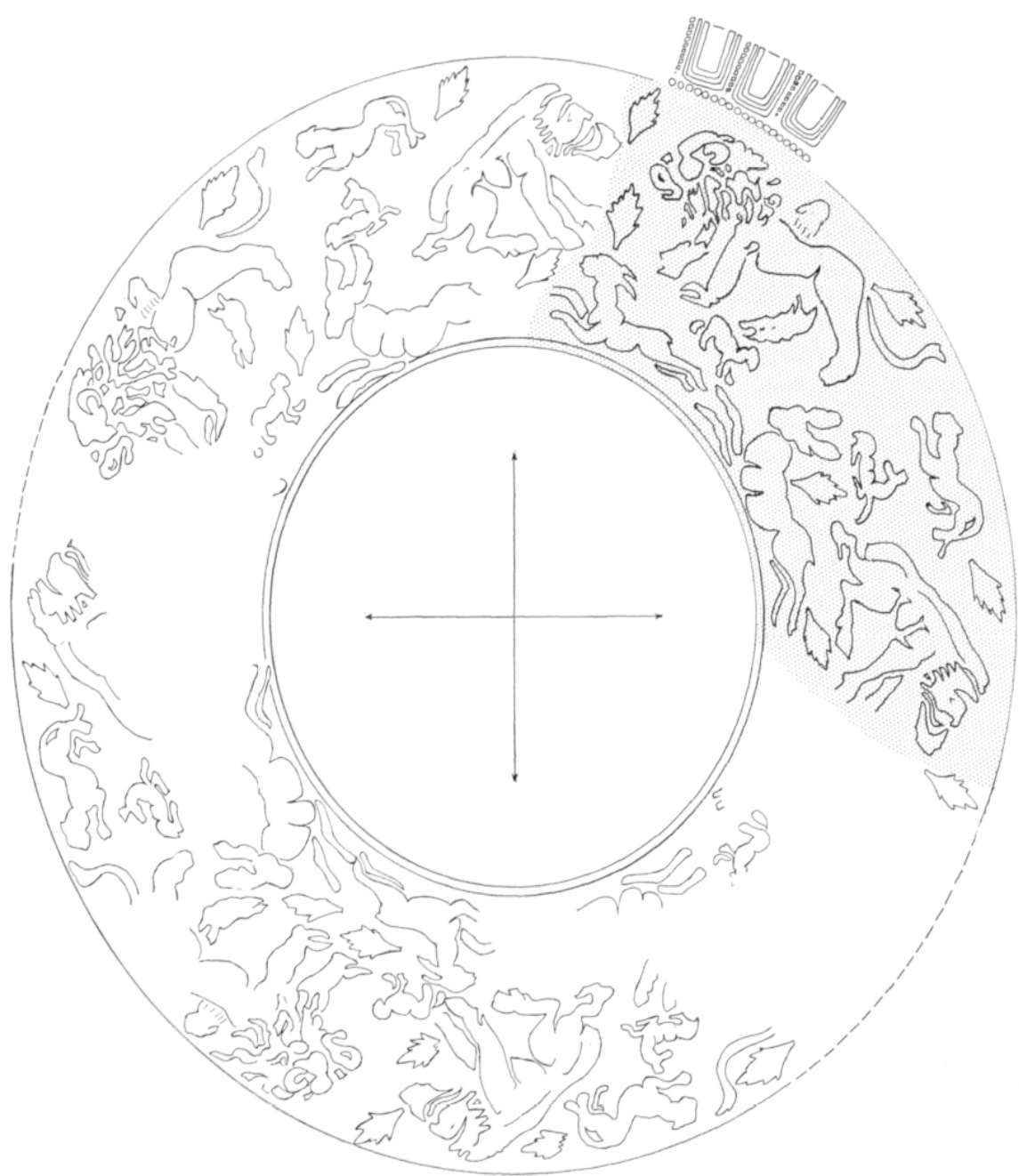

9 Schéma de décor : projection circulaire non inversée de l'ensemble de la frise du bol 65993 (en grisé et traits épais : séquence correspondant à un quart du décor, le mieux conservé?.

occupant chacune un quart du vase ; chacune contient le même groupe de sept animaux identiquement disposés - à une petite irrégularité près ${ }^{86}$ (il y a souvent plus grave, dans le style géométrique). Le rythme paraît d'ailleurs, volontairement souligné par l'affrontement, quatre fois répété, de deux animaux de taille considérable et modelés avec un soin remarquable : le lion et le sanglier. Ainsi, le style n'a de vraiment « libre » que l'apparence : la construction est rigoureuse, régulière, et se fonde sur les mêmes principes que celle du décor à métopes figuré sur la lagène : le partage en quatre de la surface à décorer et la quadruple répétition de la même séquence. Or, il ne s'agit pas là d'un accident, ou d'un exemple isolé parmi les créations de CATVSSA : Ies deux autres moules de style "libre", malgré l'état déplorable de leur conservation, peuvent témoigner - à un degré moindre, et pour cause - du même type d'organisation. Les dessins ici proposés sont des projections

86 L'un des moutons n'est pas à sa place sous les pattes du lion, mais légèrement décalé. 

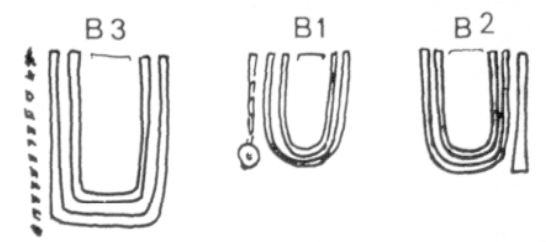

A 2

A3

A 1

00000000100

๑ロ

000000000

$\sum_{C 1} \quad 00$

000018

(2) $E 2$
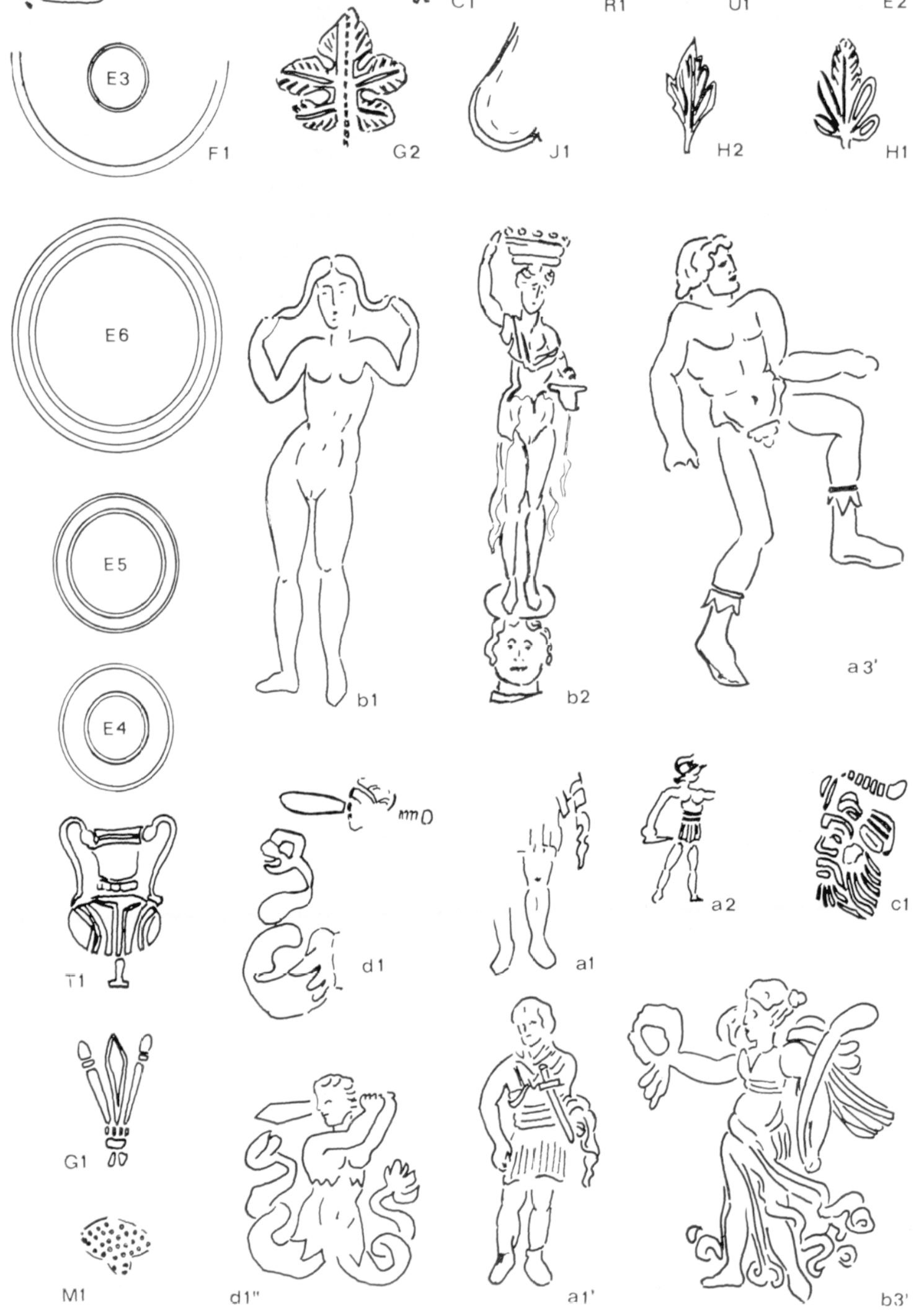


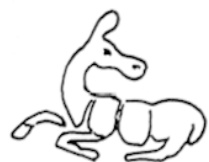

g 2

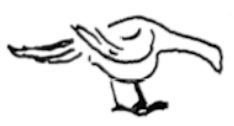

n 1
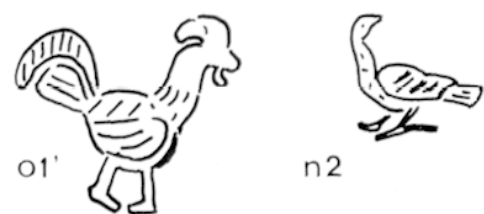

n2
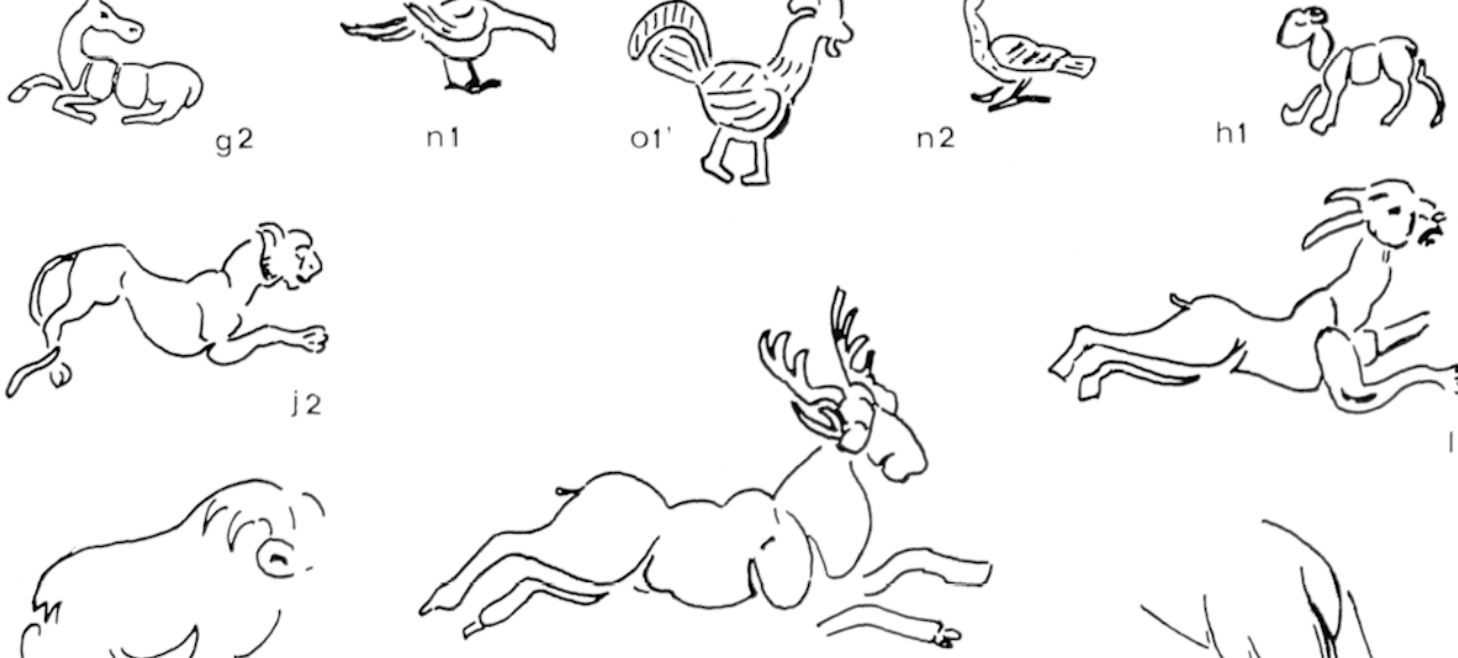

g1
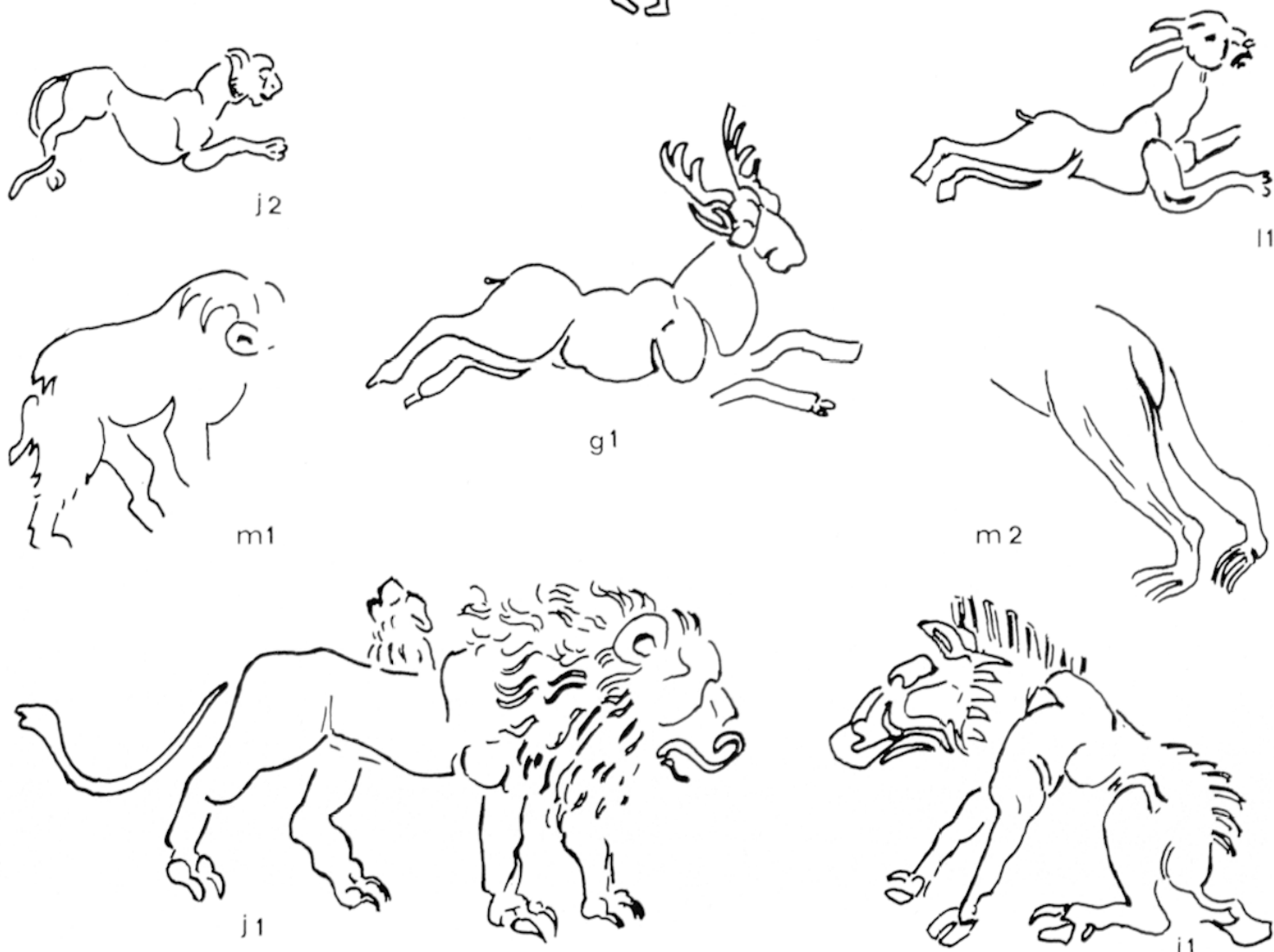

m 2
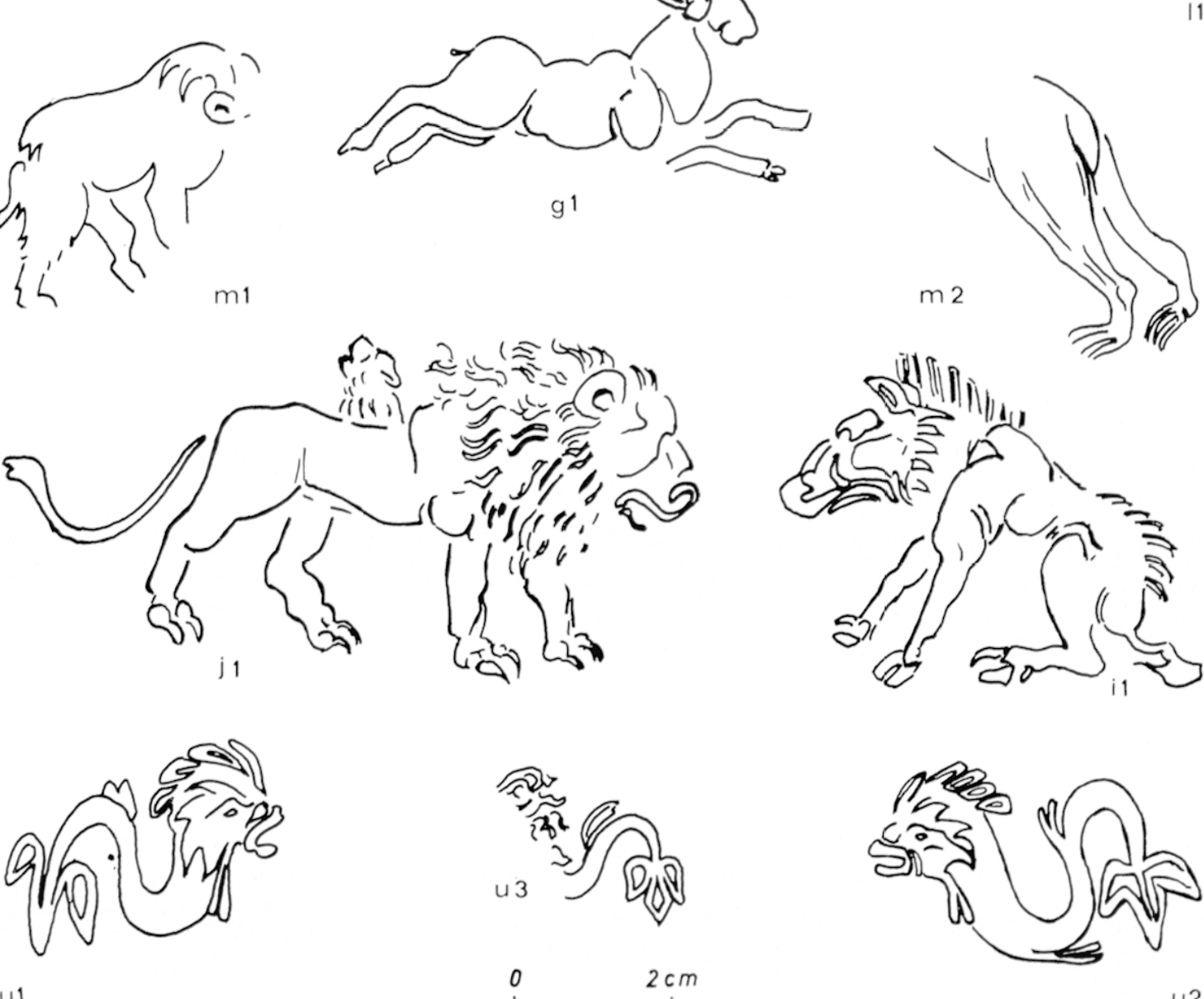

u1

$\mathrm{cm}$

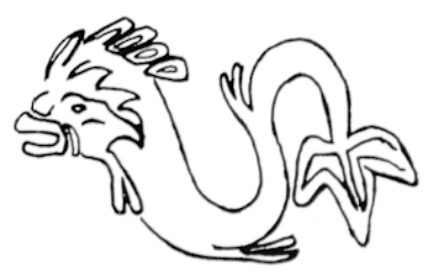

u2

10 Répertoire des poinçons sûrement identifiés et attribuables au style de CATVSSA I. a $3^{\prime}$ : chasseur (d'après le moule de CINNA.IVS) ; $d 1$ : triton (d'après P.M. 3 ) ; $d 1^{\prime}$ : motif proche complet (D. 17); a 1 : guerrier (d'après la lagène); $a 1^{\prime}$ : motif analogue complet (D. 100); $b 3^{\prime}$ : Victoire (motif complet D. 475i ; o $1^{\prime}$ : coq (motif identique et complet d'après un fragment anonyme); $m 1$ : ours (d'après le frottis) de G. Simpson). -- Tous les poinçons, à l'exception de $d 1^{\prime \prime}(=$ D. 17) et de $m 1$, figurent sur des pièces signées de CATVSSA ; tous, à l'exception de $m 1$, proviennent de relevés effectués sur des moules. 
circulaires obtenues selon le même procédé que celle du grand moule. Le fragment de décor marin (fig. 8, 4) correspond, au moins en sa partie haute, au quart de la circonférence et les extrémités du tesson conservent des vestiges complémentaires du même sujet : un triton regardant vers la gauche et brandissant des deux mains une rame figurée, en perspective, derrière la tête du monstre; le reste de la frise est composé de dauphins. De la mème façon, le moule de la seconde chasse (figr. 8, 3), conservé, en deux morceaux, sur une plus grande longueur, révèle, au milieu d'un groupe d'animaux, les jambes bottées d'un chasseur. Les deux répétitions de ce sujet se produisent - - on en a la certitude par l'ajustement exact des fragments aux cassures du fond de la matrice - à un quart de cercle d'intervalle. Si l'on compare ces restes au décor de chasse complet, il semble que l'on puisse raisonnablement estimer que les deux bols les plus détériorés étaient, comme le mieux conservé, divisés en quatre plages égales, dont la limite était indiquée par un groupe ou un personnage remarquable. De plus, le moule au chasseur prouve aussi la répétition, au moins partielle, dans deux quarts consécutifs, d'une mème séquence : on retrouve, derrière le personnage, le lion ct le bélier. Il est impossible, cependant, de savoir si cette reproduction était générale et uniforme : le haut du vase manque et la suite de la seconde séquence; en outre, l'impression tantôt d'un bélier, tantôt d'une feuille sous les pas du chasseur conduit à hésiter entre l'éventualité d'une alternance et celle d'une irrégularité : plus les motifs sont petits, en effet, plus les potiers ont tendance à les utiliser comme bouche-trous et un peu indifféremment.

Ainsi, la composition des différents types de frises témoigne principalement, quel que soit le genre choisi par CATVSSA d'un groût certain pour les constructions régulières, voire monotones. Que le style à métopes ou le rinceau prête à cette organisation soigneuse, il n'y a lá rien que de logique et de normal et la simple observation du découpage apparent a pu permettre à chacun de noter souvent, sur des vases ainsi conçus, outre le partage assez précis de la surface, la répétition constante ou alternée des motifs ${ }^{87}$. Un inventaire rapide amène d'ailleurs à constater la fréquence particulière, dans la seconde moitié du II $^{\mathrm{e}}$ siècle, de la division du décor en quatre séquences de panneaux, identiquement orientées, qu'adopte notre potier. Plus intéressante, parce que moins immédiatement apparente, semble la composition des frises "libres». Le qualificatif qu'on leur applique est, en l'occurrence, très largement démenti par la qualité de l'organisation et sans doute y aurait-il lieu, pour éviter toute antinomie, de parler plutôt de style non géométrique régulier, en réservant à d'autres cas la dénomination : style libre. La question mérite, je crois, qu'on la considère, car la technique de CATVSSA n'est pas particulière à ce potier, sans être, pour autant, générale. Cn sondage limité m'a permis de noter d'autres décorateurs, qui illustrent les mêmes principes de composition : des moules entiers de la collection Plicque, que j'ai examinés, montrent que chez ILLIXO, PAVLLVS, EPPILVS ${ }^{88}$ on

87 Cf., par exemple, H. ConforT, A central ganlish boul by Pugnus in the Walters Art Gallery, dans The .Journal of the Wallers Art Gallery, XXVII-XXVIII, 1964-1965, p. 18 ; A. P. Dersicas et F. S. A. Scot, $A$ samian boul by the potter Belsa and an arretine vessel from Canlerbury, dans The Antiquaries Journal, XI.1V, 1964, p. 155, fig. 2. Pour la théorie des types de structures, voir mon article, cilé dans li n. 82.

88 MAN 32374, 32385, 32253 (ILLIXO; ; 32382 (PAVI.I.VS); 32381 (EPPIIVS). 
divisait la surface des bols-matrices en quatre parties semblablement occupées et que le partage était souligné par l'impression d'un sujet remarquable : le plus souvent un personnage au milieu d'une population animale. Il advient, d'ailleurs, parfois, que l'on retrouve dans ces suites continues et rythmées l'alternance entre deux motifs, fréquente dans le style à métopes ${ }^{89}$. Ce qu'on observe sur les moules est reproduit, naturellement, sur les vases, et des pièces signées par PATERNVS ${ }^{90}$ m'ont amenée aux mèmes conclusions. Il serait donc souhaitable que l'on pût, grâce à d'autres inventaires, mesurer l'extension, les variations et la durée de cette mode. Les observations complémentaires que j’ai faites déjà d'après des exemplaires publiés sont, évidemment, moins certaines, dans la mesure où la projection du décor est, le plus souvent, tronconique et dépourvue de précisions sur l'importance de la fraction de frise qui a été conservée. Il est loisible, néanmoins, d'après la longueur des séries de motifs et les signes manifestes de répétition -.- intéressant soit plusieurs poinçons, soit un sujet remarquable --, de présumer qu'un certain nombre de potiers procédaient par enchaînement de séquences semblables ou identiques et pouvant correspondre à un quart du décor : on note ainsi, d'après les planches de plusieurs publications EPPILVS, PATERNTS (déjà signalés), ACAVNISSA, IVSTVS, MERCATOR II, SERVTS II et CIXNAMVS à un léger doute ${ }^{92}$; en revanche, la qualité des dessins garantit que SILVIO II et l'équipe de PATERCiLVS ont connu ce mode de construction ${ }^{93}$. Il s'agit, comme on le voit de potiers situés à l'époque d'Iladrien et à la fin du $\mathrm{II}^{\mathrm{e}}$ siècle. I'autres exemples, publiés ou inédits, prouvent, de la même façon, l'existence soit de compositions dépourvues de répétitions ou de régularité (ainsi dans la production de LIBERTVS), soit d'un style différemment rythmé. La plupart des vases ou moules de ce type, dont j'ai eu connaissance, appartiennent à des potiers clatés de la première moitié du siècle : X-3, le potier à la rosette, LIBERTVS, QVINTIIIANVS, SACER ${ }^{94}$, mais cette manière, bien que moins en faveur,

89 La limite des possibilités du sỵstème d'alternance est atteinte par des frises composées de deux séries de figures, identiques entre elles et divisées, chacune, en deux parties différant l'une de l'autre par leur contenu : le début de chaque demi-séfuence est marqué par un motif remarquable (personnage ou végetal et chacun des poingons liest répeté qu'une fois sur deux, d'un quart de vase a l'autre UAN 32387: ILIIXO); $55878:$ ALISVCIVS; $31520:$ SECVNIJINGS.

90 MAN $3187 x$.

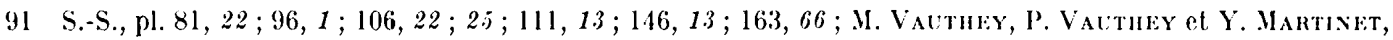
Répertoire des poingons, style el art décoratif du potier arverne SERVVS II, dans Rev. arch. du Centre, 23, 1967, pl. XIV. Tous ces potiers sont datés apres $135 / 140$.

92 La répétition est visible, mais le rythme incertain pour BVTHIC) S.-S., pl. 59, 662; 58,656;61, 681;.

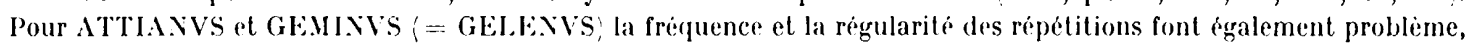

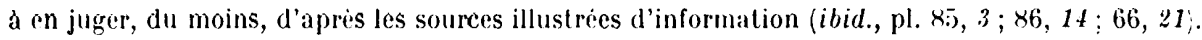

93 l)ans le cas de ces dessins 00 . cit., pl. 77,1 et 72,38 ) les decors sont mis en place par rapport au pied du vase, lui-même figure. Il n’echappe pas, ainsi, que le même groupe d'animaux (lion terrassant un sanglier se répete à un quart de cercle d’intervalle sur le bol de SII.VIO II et que la composition attribue à PATERCI.VS etait divisée en quatre séquences presque identiques (un des petits guerriers varie, indiquées par le relour regulier de deux grands gladiateurs affrontés.

94 Ibid., pl. 14, 173: très longue séquence sans répétition visible, égale à la moitie du décor ou plus grande (?); pl. 26, 319 : répétitions bien apparentes, mais la longueur de la séquence, par rapport a la hauteur de la zone ornée, et son caractère continu semblent exclure une division en quatre; pl. 51, 601, 603, 622 : on note surtout l'extrême diversité des motifs ; pl. 68, 7 : répetition de quelques peinģons d'une moitié de vase à l'autre, sans qu'aucune division soil visible dans la moitié complète qui subsiste, et la régularité de l'ensemble, du fait des lacunes, n'est pas assurée; pl. 82,1 : longue suite dont l'extension et l'organisation interne nous echappent. 
parait survivre encore, à une époque plus tardive ${ }^{95}$. Il ne s'agit là, c'est l'évidence, que d'une brève enquête, nécessairement limitée par le hasard des découvertes, l'état du matériel, la rareté des publications, et aussi les intentions de ce travail. Mais il importe de constater quelle a mis en évidence plusieurs styles "libres", que tous sont non-géométriques, certains réguliers, et que, au premier abord, ces différentes modes ornementales semblent n'avoir pas eu la même durée, ni la mème extension. On peut, dès lors, souhaiter de voir préciser et nuancer ces observations par un recensement plus complet des ateliers qui ont adopté l'une ou l'autre de ces manières, et par une étude de la compatibilité de deux ou plusicurs de ces techniques, dans une même production, ou, au contraire, de leur caractère exclusif.

Il apparaît, au terme de cette analyse, que les moules de CATVSSA appartenant à la collection Plicque trahissent, à travers la richesse du répertoire (fig. 10) et la diversité des thèmes et modes de décoration, une réelle unité de conception. Proches, dans l'ensemble - par certains motifs, ou par la technique un peu sèche - de modèles de la seconde moitié du ${ }_{I^{\mathrm{e}}}$ siècle, ils se caractérisent pourtant, souvent, par de très remarquables rapports avec le répertoire ou les procédés des premières décennies. De plus, certains éléments tenus pour spécifiques de la fin du siècle, comme les grands médaillons ou les arceaux sont à peine utilisés. Comme, néanmoins, de tels poinçons apparaissent sur d'autres produits du potier, il est raisonnable de penser que la manière du décorateur a pu varier, en particulier selon les différents moments de son activité. Enfin cette similitude générale des principes que nous cherchons ici à nuancer, n'exclut pas une notable discontinuité : plus que l'originalité - relative et prévisible - de la valve de lagène, il faut souligner la situation à part du vase à la frise marine. Toutefois, il est permis de supposer qu'un échantillonnage plus complet des vases de CATVSSA rendra compte de cette diversité : nous pouvons actuellement l'imputer à de multiples raisons -.. les plus simples étant le hasard, qui a guidé la fabrication des pièces que nous connaissons, et l'intervalle séparant dans le temps les créations d'un même atelier. Tant il est vrai que la similitude, grossière, des signatures et la parenté, générale, des décors entretiennent la conviction -.. peut-être illusoire - que toutes ces pièces ont une origine commune.

Plus délicat paraît le problème posé par l'existence d'un second groupe - constitué principalement, pour l'heure, de vases - signalé également à l'attention par la signature: CATTSSA. Il se différencie, dès l'abord, du premier par l'emploi d'estampilles intradécoratives (fig. 11, II, a-e). L'examen des quelques échantillons qui m'ont été accessibles a permis de déterminer leurs caractéristiques et je me bornerai à résumer les principales observations à verser au débat. Les décors relèvent pour une part du style géométrique, pour l'autre du style dit libre. Les cinq tessons que j'ai vus (fig. 12), ou dont on m'a communiqué la reproduction ${ }^{96}$, comprennent un exemple de grand rinceau, trois de

95 Cf. vase d'Albvcivs (150-190 S.-S., pl. 123, 42) : le fragment, qui correspond à peu de cheses près a la moitié du vase, paraît comporter à ses deux extrémités les restes du même cheval, mais aucune séparation interne du groupe d'animaux n'est sensible.

96 Deux tessons appartiennent au Musée des Antiquites nationales (MN 66521 ol 65521), le troisieme a la collection Fabre, de Clermont-Ferrand, les derniers, aux musées de Dieppe et de Rouen. Je dois à G. B. Rogers le 

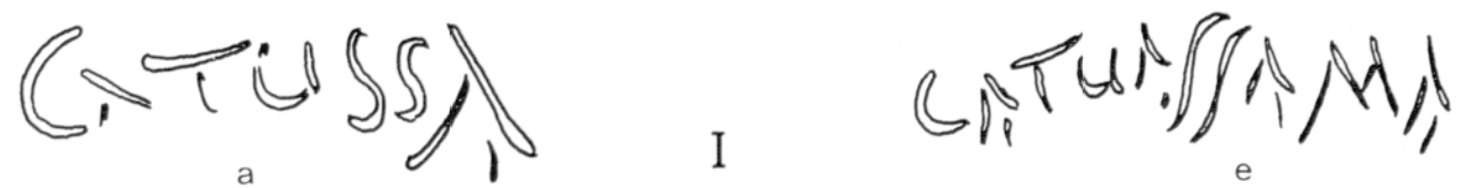

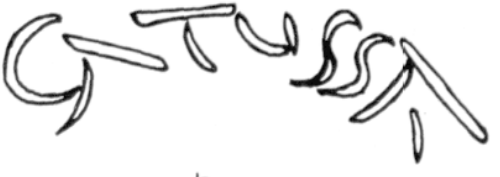

b

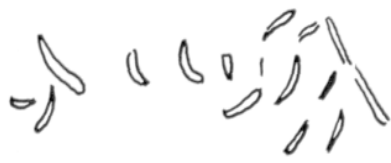

C

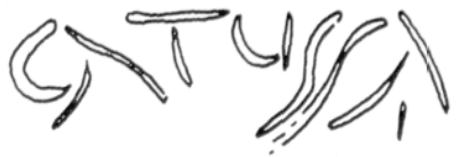

d

$(600000000$

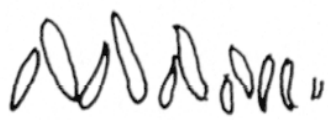

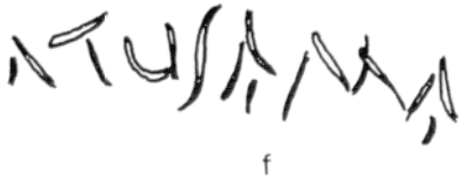

$\ln _{10} \mathrm{n}$

9

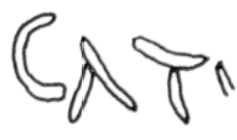

h

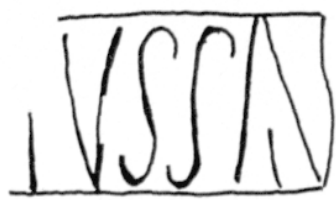

\section{CTTISS,}

11 Signalures. I : attribuès à CATVSSA 1. $a:$ P.X. $2 ; b: 663 \% 0 ; c:$ P..1. $1 ; d: 65993 ; e:$ l.ezoux $1962 ; f:$ Lezoux

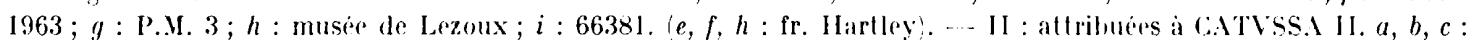
issues probablement du mème poinçon Rouen, $1 \mathrm{AX} 6552 \mathrm{l}$, lleppe ; $d, e$ : issues peut-ètre du mème poinçon plus ou moins use (Clermont-Ferrand, MXN 66521); $f$ : graffite de Colchester ?? . $d$, f : fr. liogers pt Hartley - Toutes les signatures en relief (provenant de vases) sont reproduites en traits noirs pleins; normalement retrogrades, elles ont été ici inversées. Les autres, en creux, proviennent de moules. 
compositions à métopes, un de chasse et le répertoire identifiable (fig. 13) comporte une vingtaine de poinçons ${ }^{97}$. L'ensemble du groupe, à la différence du précédent, semble homogène dans la mesure où les mêmes détails secondaires sont uniformément utilisés : tous ont les mêmes cordons de séparation et l'ove, conservé quatre fois, est toujours identique et, actuellement, propre à cette série (fig. 13, B1). L'exiguïté des morceaux limite les remarques que l'on peut faire sur le style du décorateur. On constate, néanmoins, que les frises géométriques (fig. $12,1 b, 3,4$ ) se rattachent très exactement à la mode de la fin du II $^{\mathrm{e}}$ siècle, en particulier par le nombre des demi-cercles et des très grands médaillons; la même observation s'impose à propos du rinceau (fig. 12, 2), très proche autant qu'on en puisse juger -- des créations de PATERNVS ou CINNAMVS, par exemple. En outre, CATVSSA imprime, à la manière de l'époque, de grandes estampilles et s'efforce, comme le font PATERNVS, SERVVS II, CINXAIVS, BANVVS, d'encadrer sa signature, en la substituant au contenu décoratif d'un médaillon ou d'une métope. Enfin, le catalogue des poinçons identifiés n'a, pour l'instant, aucun point commun avec celui des moules de Saint-Germain, ni, apparemment, aucune parenté significative avec celui des moules de B. Hartley; bien plus, CATVSSA II use aux mêmes fins que CATVSSA I de poinçons différents : ainsi la rosette (fig. 13, C 1) utilisée comme motif de remplissage dans les trois frises est propre à ce groupe. La même concurrence s'observe plus nettement encore sur le vase de la chasse $($ figg. 12, $5 b)$ : le décor est conçu selon un mode particulier - avec: des arbres --; le semi du fond est totalement différent de relui de la première série; le mème animal - le bouquetin (?) - - est figuré, dans la même attitude, à l'aide d'une matrice différente (fig. 10, 11 et 13, 11). Done, l'auteur, s'il s'agit du décorateur de nos moules, aurait changé délibérément son répertoire. Dans ce cas encore, les rapports avec des ateliers tardifs sont extrèmement nets : le tortillon (fig. $12,5 b ; 13, \mathrm{l}: 1$ ) dans le décor libre rappelle PATERNVS et CALETVS-SEVERVS98, l'arbre (fig. 13, N1) est un poinçon de CINNAMVS ${ }^{99}$. Cet ensemble d'échantillons parait donc parfaitement distinct du premier, en même temps que techniquement uni et chronologiquement homogène. Devons-nous le séparer radicalement de nos moules? Lin tesson, dont B. IIartley m'a très aimablement communiqué le frottis (fig. 12,6), montre que l'hésitation est possible, voire recommandable : ce fragment de bol, trouvé en Angleterre ${ }^{100}$ révèle l'emploi, par le créateur d'une chasse dans le style de CATVSSA II, d'une signature manuscrite infradécorative. L'inscription (fig. 11, II, f), assez largement conservée, s'apparente en une certaine mesure (par la forme de l'V en particulier) à une petite capitale. Le répertoire, réduit et composé

dessin du fragment de Clermont-Ferrand. Les échantillons de Haute-Yormandie m'ont été aimablement communiqués par les conservateurs des musées de Rouen et de Dieppe, Iflle Chirol at M. Bazin (voir, pour les signatures des vases normands, deux articles de .I. MaxgarD, Estampilles de potiers galln-romains, dans Bulletin des amys du vieux Iteppe, LXX, 1964, p. 6:-64 et LXXVI, 1969, p. 29-32).

97 Eros : 0.401 et 449 ; Bacchus : 0.557 ; biche: 0.1805 ? ; lionne : 0.1533 ; bouquetin? : 0.18 .42 ; petit cerf : 0.1785 ; arbre : $\ 8$; motif géométrique : 971 ; rosette : $: 58$; feuille : $11100 ;$ ore : B 1.41; cordon : A 2 . A celte série s'ajoutent des cercles, arceaux el tortillons non répertoriés.

$98 \quad$ S.S., pl. 106, 19, 21, 22, 24; 128, 2, 5 .

99 Ibid., pl. 163, 72 et fig. $47,20$.

100 L'origine exacte de la piece : Colchester, n'est que présumée. 
pour une part de poinçons peu connus (figr. 13, g2, g4, j1, m1, L1), est, en tout cas, complètement différent de celui des deux moules de CATVSSA I précédemment étudiés ${ }^{101}$.

Ce bref inventaire révèle donc que l'ensemble des échantillons considérés tend à s'organiser en plusirurs séries. Deux d'entre elles sont nettement différenciées par la qualité de la signature et la nature des motifs décoratifs : celle de la lagène et des graffites cursifs; celle des marques estampillées. La troisième parait correspondre à un groupe intermédiaire, signé sous le décor, à la main avec une écriture un peu différente de la rursive. In fait, les limites de ce dernier sont encore mal définies dans la mesure où ses caractères demeurent, pour une part, négatifs et où son homogénéité ne se fonde, actuellement. que sur une certaine analogie du zraphisme des signatures, tracées à la main et imparfaitement conservés. La prudence commande done de réserver son jugement sur la parenté des trois fragments, mais de retenir le résultat essentiel de ces analyses, qui est de révéler l'hétérogénéité et du matériel attribué à CATYSSA I, sur la foi des graffites, it de la série relevant du style de CATYSSA II : le premier semble employer différents types d'écriture et un répertoire très diversifié, le second, plus cohérent, sans doute, dans sa manière, signe tantôt à la main, tantôt à l'aide d'un poinçon.

Au terme de cette éturle partielle, il semble que l'ensemble des produits signés CATVSSA, auxquels j’ai eu acè̀s, soit conforme aux modes de l'époque des Antonins, et principalement des derniers d'entre cux. L'analyse stylistique appliquée au détail d'un certain nombre d'échantillons ... des moules surtout .... a permis, en effet, de dégager, tant dans le style dit libre, que dans les décors à métopes, des principes de compositions communs à un certains nombre d'ateliers à partir du règne d'Hardrien et illustrés plus encore après 150 : nous trouvons là une confirmation globale des observations de G. Simpson, dans la mesure, du moins, où l'analogie avec le style de potiers par ailleurs datés permet de proposer une chronologie. Ces considérations, fondées sur des raisons esthétiques sont, d'autre part, en accord avec les déductions dont B. Hartley nous a fait part et auxquelles il a été conduit par un inventaire des signatures, tant en GrandeBretagne qu'en France, et à la suite de découvertes récentes dans les fouilles de Lezoux. Cess conclusions paraissent raisemblables, mais demeurent très générales : ainsi le problème du rapport entre les deux CATYSSA (ou supposés tels) n'est ici qu'incomplètement abordé, puisqu'il na été envisagé que comme complément de l'étude des pièces d'une collection. In examen attentif a cependant permis, d'après ce matériel limité, de nuancer les hypothèses auxquelles pouvaient prêter deux séries apparemment distinctes. En effet, certains aspects du style des moules Plicque ont suggéré l'existence de relations étroites entre le répertoire de LIBERTYS et BVTRIO et celui de (iATVSSA, puisque les mèmes poinçons ont été utilisés par ces potiers (la feuille, la Vénus, la caryatide, un oiseau -pour ne citer que ceux que j'ai eu les moyens de contrôler), et incité à envisager l'influence possible de diverses manières décoratives du début du siècle sur la technique de notre potier. Ce sentiment nous a été inspiré, nous l'avons vi, par l'analyse du décor de rinceau et, indirectement, par l'attribution primitive d'un vase de CATYSSA, de style libre, à

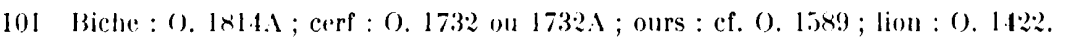



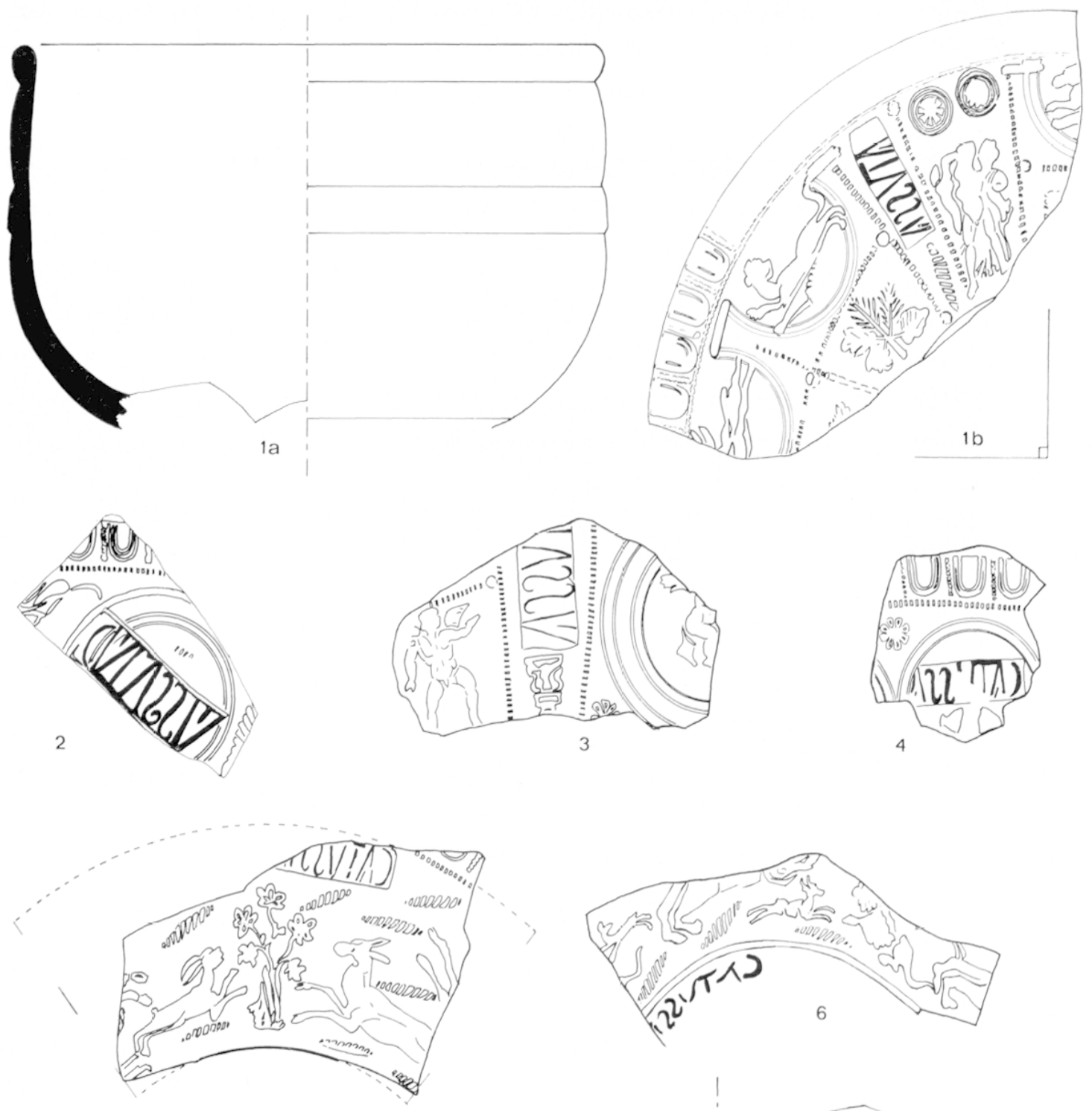

$5 \mathrm{~b}$
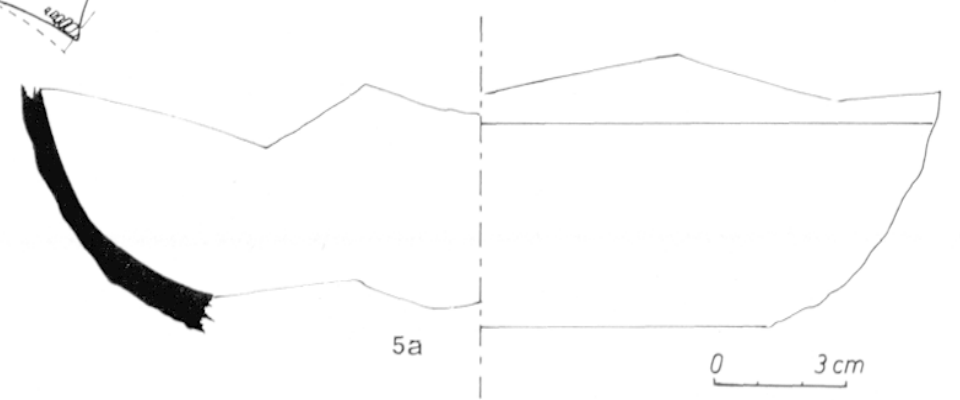

12 Vases attribuées a cATVSSA JI. 1 a : coupe of profil du tesson de lieppe; 1 b : projection circulaire du decor

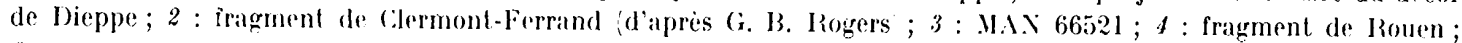

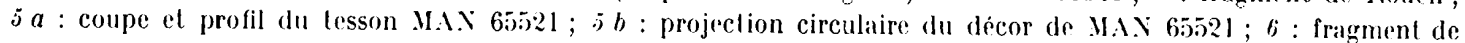
(Colchester ?) (d'apres B. Iartley: 


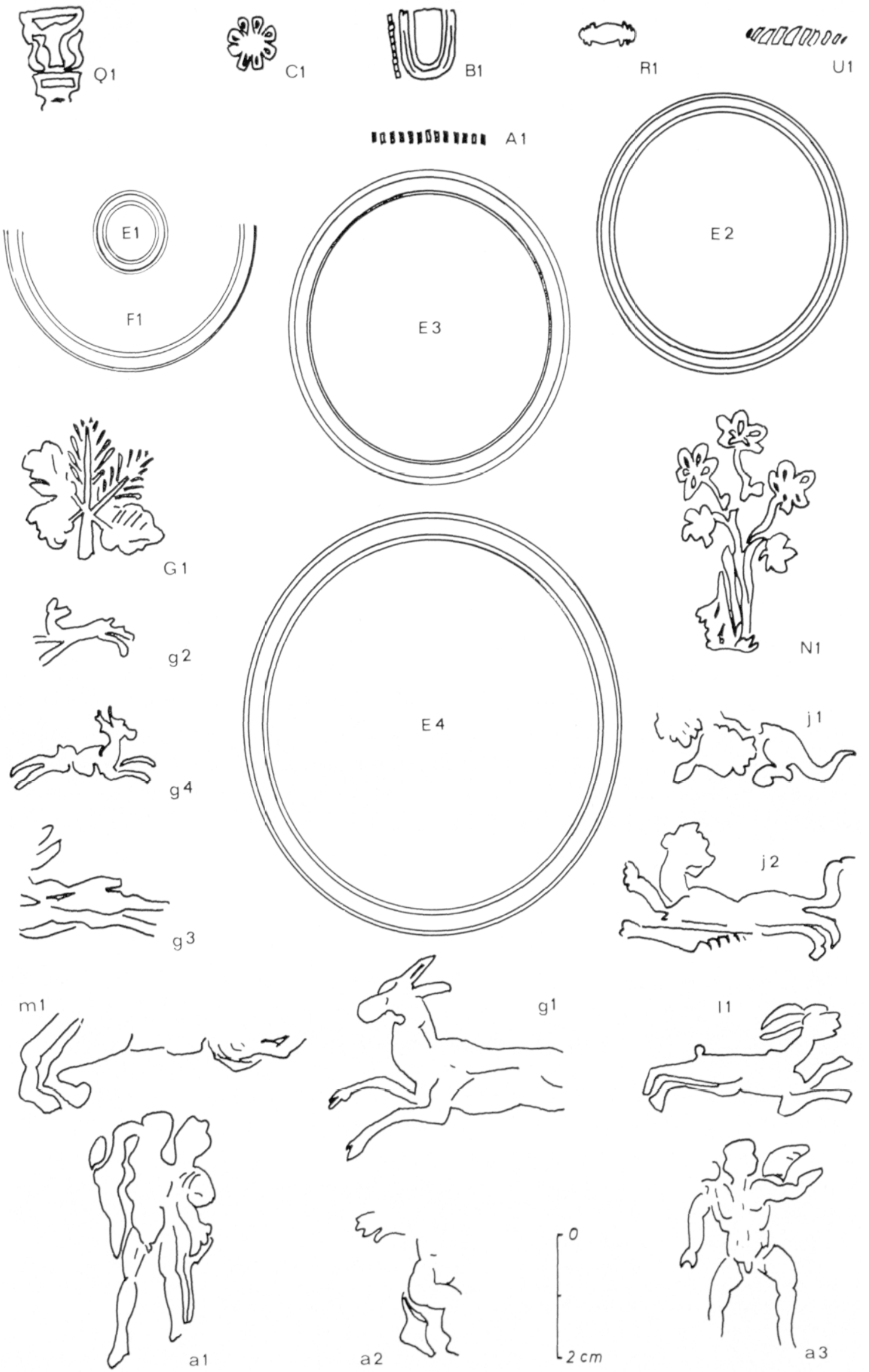


l'atelier de BVTRIO. Les études stylistiques sont, à l'heure actuelle, encore trop peu avancées et ce matériel est trop réduit pour qu'on déduise, à coup sùr, de parentés esthétiques, une datation précise, et plus haute des débuts de CATVSSA (vers 135/140?), plutôt que l'irlée d'une éventuelle survivance. Toutefois la question reste posée deś conditions d'utilisation commune ou du morle et de la date de transmission, de LIBERTYS-BITRIO à CATYSSA, de modèles rares et, par ailleurs, peu attestés parmi les répertoires de la fin du $\mathrm{II}^{\mathrm{e}}$ siècle.

D'autre part, l'étude des vases estampillés dans le décor a fait apparaitre un ensemble, pour l'instant, cohérent et spécifique et montré l'incontestable dépendanee des diverses techniques de composition (métopes, rinceaux, ('hasses) par rapport au style des décennies 150-190 le plus caractérisé. Enfin, chacune de ces familles contenait, nous l'arons constaté, des éléments allogènes, comparables, au moins pour deux d'entre eux (le vase de Colchester attribué à CATTSSA II, un moule de Lezoux à grands médaillons), aux produits du ${ }_{11} \mathrm{e}^{\mathrm{e}}$ siècle final ${ }^{102}$.

L'alternative se présente, à nos yeux, de la façon suivante : il a existé deux CATTSSA; l'un reflète, par une part de sess créations, une influence des ateliers des premières décennies, l'autre reproduit de façon asserz stéréotypée des poncifs de la seconde moitié du siècle. Dans cette hypothese, la question n'est pas encore tranchée de savoir si tous les moules ou vases, tenus actuellement pour allogenes doivent, comme l'un d'eux, relever de la production de CATVSSA II. Ce dernier, en tout as, a pu ne pas exereer toujours ses talents au même titre : l'usage tantòt de la signature infradécorative, tantôt de l'estampille intradecorative pourrait, par exemple. se justifier chronologiquement et supposer comme le pense. II. Lutz a propos d'autres ateliers ${ }^{103}$ - le passage du statut d'employé (ou de successeur) a celui de patron. Si l'on admet, comme (a. Simpson en réserve l'éventualité, qu’il na existé qu'un CATYSSA, on devra alors expliquer la qualité variable des signatures et la diversité du répertoire : nous avons remarqué, en effet, que le même type de décor (les (hasses) comporte deux séries de poinģons distinctes, alors que lessujets figurés sont les mèmes. Faudrait-il voir là la trace des différentes officines qui, surcessivement, auraient employé cot artisan jusqu’à ce qu’il devint, chef d'entreprise? Scule. varisemblablement, la connaissance de nouveaux documents permettra de choisir, et de resoudre ce dilemme.

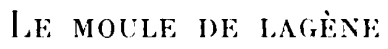

La value de lagène (fig. 1, j), tronconique et légerement galbée. ast faite d'une pâte rose, micacéc, soigneusement lissée. Ie poli de la surface intérieure et la fraicheur du modelé des décors ne permettent pas de penser que ce moule, de facture soignée, ait beaucoup servi. Les grands fragments conservés attestent que Io diametre intérieur était

102 Cf. en particulier lo moule aux dauphins de lezoux.

103 Latelier de Sallo el de Salurninus à Willelbronn (Moselle), XXlle supp. a ciallia, 1970, p. 193-194. B. Hartley m'a signale l'emploi conjoint de deur signatures sur deux echantillons de I.ezoux : CATVSSA, graflite infradecoralif, et une estampille non identifiee dans le decor, sans preciser, toutefois, s'il s'agit du style dit de C.ATVSSA II. 
d'environ 22 cm. La hauteur totale nous est inconnue, mais dépassait $15 \mathrm{~cm}:$ la zone qui subsiste atteint $14 \mathrm{~cm}$, ou peu s'en faut, et le registre du haut est incomplet ${ }^{104}$. Yous ignorons, du fait des détériorations, quel était le profil de l'orifice supérieur ; nous voyons, en revanche, que la base du moule s’épaissit extérieurement d'un bord vertical, dont la hauteur varie, sur les tessons. entre 39 et $13 \mathrm{~mm}$, selon une pente insensible.

L'inventaire des lagènes - moules ou vases - établi récemment par L. Balsan et A. Vernhet ${ }^{105}$ a montré que de nombreux vases de ce genre sont attestés a la Craufesenque et que la plupart des exemplaires signales par des publications proviennent d'ateliers du Sud de la Ciaule, les plus tarlifs etant actuellement datés de la fin du I $^{\mathrm{er}}$ siè le ${ }^{106}$. Cette forme, cependant, était produite aussi dans le centre : cest, en effet, a partir de cruches vernies de saint-Rémy-en-Rollat que J. Derehelette etablit l'existence de son type 6:107. I)'autre part, des eruches moulées ont pu être fabriquées à L'zoux : il s'agit non seulement, de petits vases analogues à ceux de Vichy et représentés, dans la collection des moules de Saint-Germain, au moins par un tesson de valve supérieure, mais aussi de pièces sigillées comparables à celles de la Ciraufesenque. Lin effet. des fragments de matrices décorées par zones ont èté signalés réeremment dans le matériel de Lezoux ${ }^{108}$ : l'un, autant qu'on puisse juger d'après le cliché, pourrait être un morceau de valve inférieure; l'autre appartient, sans aucun doute, à une valve supérieure. Ils ont été découverts dans les fouilles d'un four, sont attribués à TITOS et clatés vers le milieu du r $^{\text {er }}$ siecle. Par ailleurs, le tri du matériel de la collection Plicque n'a permis d'isoler d'autres restes de pièces ovoüdes ou tronconiques, décorees par bandes et qui pourraient avoir servi à mouler des cruches.

Le premier tesson (fig. 14, A) conserve, sur une faible hauteur, le bord d'un moule orné d'un rinceau (très endommagé) à feuilles palmées ${ }^{109}$, sous une rangée d'oves à un seul arceau et à baguette lisse. Le profil. pour la panse, fait largement défaut. mais la mouluration du bord (fig. 14, :11) distingue. sensiblement cette matrice d'autres moules ordinairement creusés d'une gorge intérieure en leur partie supérieure -.. ceux des I). 29 en particulier : il s'agit, en effel, en la circonstance, d'une entaille profonde. qui présente deux faces rectilignes et orthogonales; l'une est parallèle au profil extérieur du fragment et, l'autre, évidemment, perpendiculaire ì ce dernier. Cette découpe. comme je l'ai vérifié, est tout a fait analogue à la feuillure (fig. 14. B1 et B2) signalée par Hermet au bord de certaines valves de lagienes ${ }^{110}$ et paraît n'avoir de nécessilé autre que de permell re l'emboitement

104 Ces dimensions impliquent que la partie superieure du vase, comple temu de la retraction, avait des proportions comparables à celles de deux lagenes de SABINVS, que j'ai pu voir et mesurer, gràce à l'amaluilité de L. Balsan

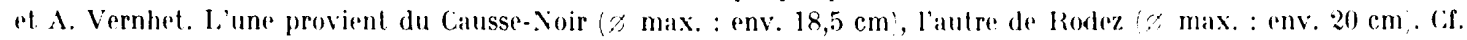
J. A. Staxitrin, Romano-gaulish decoraled jugs and the trork of the potler Sabinus, dauns Journal of Roman Studies,

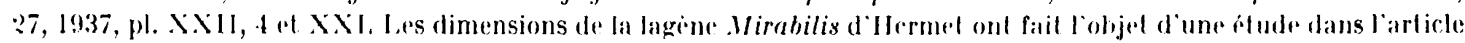
citc ci-apres : cette piece entrait ígalement dans la même cal iggorie.

I05 I'ne grande lagjene de la Ciraufesenque, dans Ciallia, XXIX, 1971, p. 7.1-75.

106 les 130 exemplaires recensés proviennent pour la plupart de la Graufesempue, mais yuelyues-uns sont signales à Nontans at a Bamassac. Ja datation la plus haule est celfe qu'on propose pour un vase de Neuss de type II. 15 (G. MARY, Drei Terra-sigillata Kräge alus Neluss, dans Bonner Jahrhücher, 1959, p. 178 ol pour une cruche de

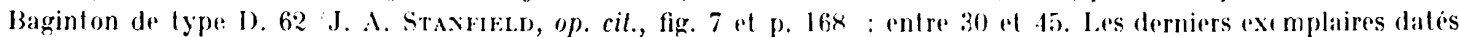
sont considerés comme flaviens, voire un peu plus tardifs.

107 Op. cil., I, p. tx.

lox Informalions, dans firallia, XXV, 1967, p. 321, fig. 41 : lorientation du moule de droite coves en bas est indiqués par les personnages ; pour determiner sùrement la nature du fragment de ganche il faudrait en connaître exactement la forme : en tout cas, le decor en bandes, arec oves, ne comporte pas de tace depaulement.

109 J'emploie, par commodite, la terminologie d'Hermet.

110 Op. cil., pl. $116,3 b$. 

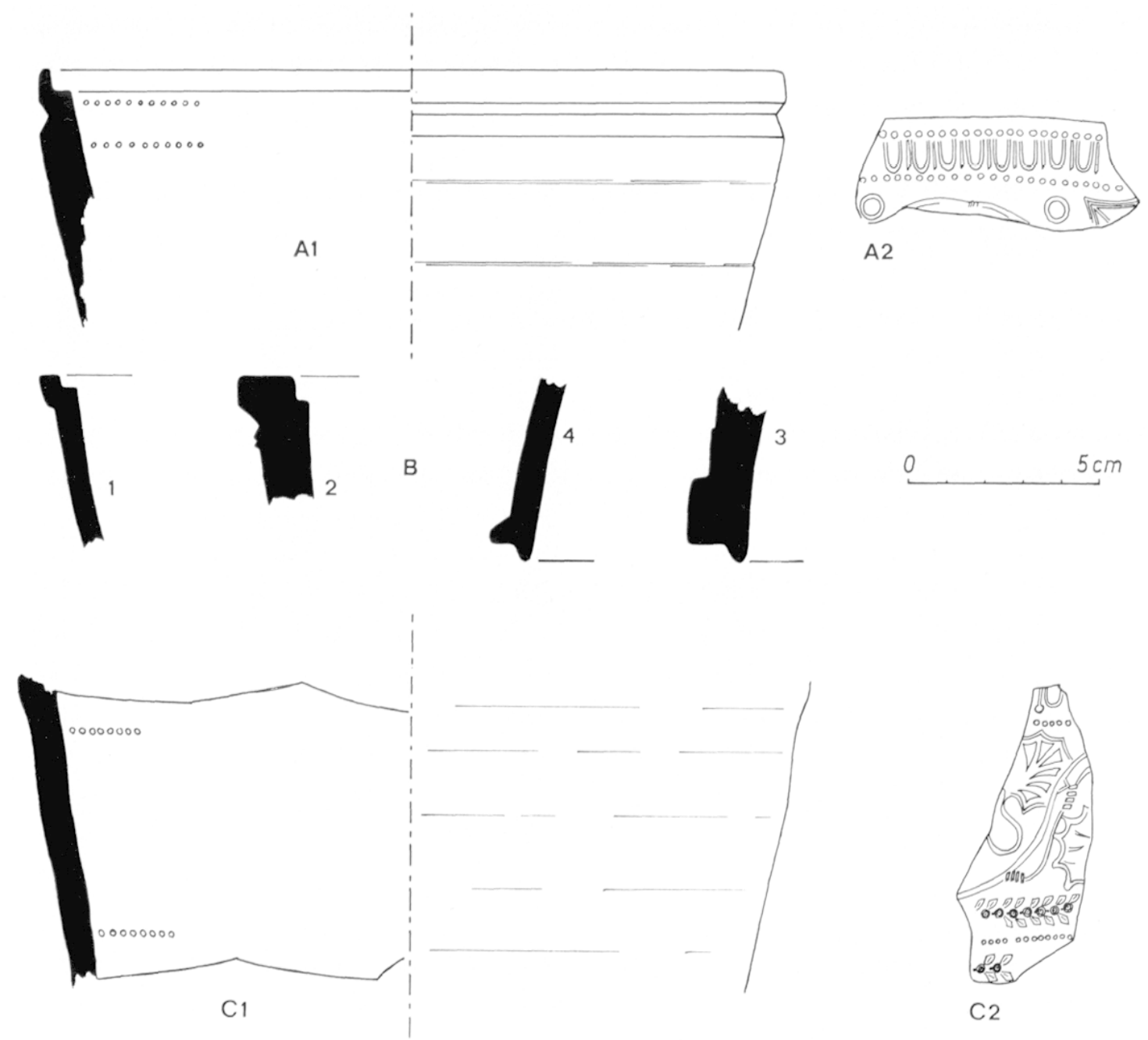

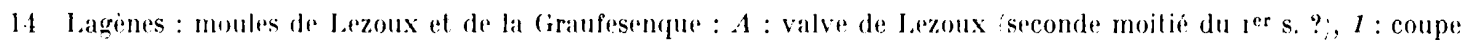

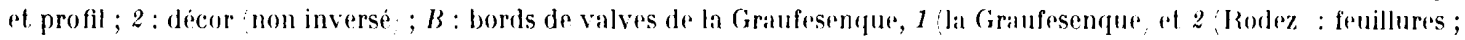

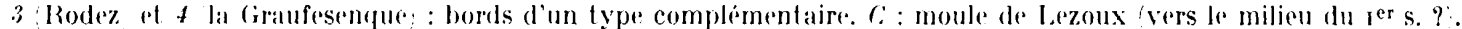
1 : coupe et profils ; 2 : decor'non inverse . - L'orientation de $A(1,2), B(1,2), C:(1,2)$ est arbitrite, compte tenu de l'élat et du decor des exemplaires reproduits.

d'une pièce complémentaire (fig. 14, B3 et B4). I)'un point de rue typologique, en tout cas, aucun autre modèle. actuellement publié, que la lagène ne réunit les mèmes caractères : le rinceau est. compatible avec le décor par zones d'un I). 29 mais la lionne d'oves exclul cette identification; de plus, la moulure des 1). 29 se présente, d'ordiniire, comme une dépression aux lignes douces, profilée en are de cercle. La disposition de l'ornementation en bandes superposées, terminées par une ligne d'oves est parfois celle des calices I). 11, mais si les rares exemples de matrices signalées el figurées ${ }^{111}$ sont souvent bordés de gorges, je n'en connais pas qui comporte une feuillure. Or la valve de lagène se caractérise, au moins dans certains cas, par un bord entaillé, un décor à registres superposés et une bordure d'oves.

111 Voir entre autres, F. H:rmet, ibid., pl. 116 ; H. Vertet, Céramique sigillée Tibérienne de Lezoux, dans Rev. arch., 1967, p. 255-288, fig. 7, 12, 17, 18, 22. 
Le deuxième tesson (fig. 14, C) appartient à la mème catégorie de moules. I'un brun plus soutenu que le premier, il est. comme lui, soigneusement lissé et orné. Et il semble que seule une matrice de lagène puisse concilier toutes les particularités du fragment, malheureusement très petit et ne conservant aucun reste de bord, ou --- éventuellement -.. de fond. Le profil, d'après les traces de tournassage, est incontestablement tronconique ; le décor est organisé par zones et celle qui correspond au plus grand diamêt re conservé est constituée par des oves. De plus, un épaississement marqué de la paroi, au niveau de cette frise, autorise à croire qu'on se trouve a proximité d'un bord. Parmi les moules, publiés ou inédits, auxquels j'ai eu accés, seules des valves de lagrines unissent à un profil en Ironc de còne un décor en bandes, bordé d'oves. Les regristres de notre fragment de matrice se succèdent ainsi (fig. 1.1. C2) : une ligne d'oves a un arceau arec une baguette lisse terminée par un pendentif circulaire; puis. apris, une rangée de petites perles, un rinceau de feuilles palmées aux tiges tròs sinueuses, tracées a la main ; enfin, de part et d'aulre d'une seconde ligne de perles. deux étroites guirlandes de fleurons (constitues de feuilles ovales et pointues, opposées deux à deux et se rejoignant dans un cercle pointé. lui-mème suivi d'un petit point \%.

Compte tenu de l'étal des tessons el de la rareté des publicalions. pour ce genre de matériel. il parail malaisé de pousser beaucoup plus loin l'analyse. Il est bon de noter, cependant. que la qualité des moules et la nature des décors sont tout a fail compalibles avec ceux de matrices de lezoux du I $^{\mathrm{er}}$ siecle le12. En effet. des oves du mème genre que ceux du premier fragrment apparaissent dans le dépotoir tibérien réremment découvert ${ }^{113}$ et se retrouvent associés à un rinceau de feuilles palmées, analogrue aux nòtres, dans un décor silué entre 40 et 60 et ornant une valve supérieure de lagìne114; enfin un type de fleuron tout a fait semblable a celui que nous avons vu figure sur les tessons d'un moule I). 29, de même origine que la valve el portant la signature du potier TITOs. La question subsiste, toutefois, pour dater nos deux fragments de connaître la durée, à Lezoux, de tels éléments de décor ${ }^{115}$. Il reste, d'autre part, a savoir quelle partie du moule a été conservée: les oves n'offrent aucun repire, puisque leur limite supérieure rectiligne est, normalement, alignée le plus pris du bord du moule; les rinceaux et guirlandes ne permettent pas davantage de choisir une orientation. Le profil ne nous aide pas plus : il est conservé sur une très faible hauteur et, d'autre part, la ty pologie de tous les modèles de lagènes reste à faire. La forme de la feuillure, enfin, n’apporte pas d'arguments décisifs : il existe, en effet, à la Graufesenque el à Rodez ${ }^{116}$ des matrices bordées d'une moulure en saillie et propre à s'encastrer, comme le montre Hermet, dans une feuillure ${ }^{117}$, et les deux échantillons que j'ai vus (fig. 14, 133 et B4) proviennent logiquement de valves supérieures ${ }^{118}$. Peut-on en conclure que tous les moules à feuillure étaient des valves inférieures" Ce serait prémaluré : le nombre des pièces inventoriées est insuffisant; de plus, les deux fragments de bords a feuillure que j'ai vus a la (iraufesenque et au musée Fenaille ne fournissent aucun indice propre à déterminer

112 Il s'intigre dans une série soigneusement el finement décorée, aux parois minces et bien lissées, tournée dans une pàte gris-brun ou jaunâtre, fine, micacée.

113 Op. cil., p. 266, fig. 4 (1-5).

1.14 Informalions, dans Gallia, loc. cil.

115) I.e décor de notre premier fragment, à la difference de celui qu'on at tribue a Titos ef de celui de notre second tesson, est compléte par de petits anneaux, qui occupent les blancs entre les tiges of les feuilles. Ce type de rinceau, avec different motifs de remplissage, apparaît déja sur les premieres lagenenes de la ciraufesencque, où il se

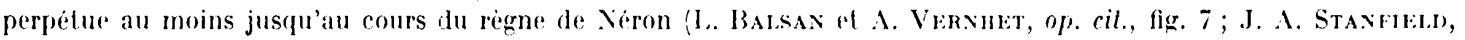
Romano-gaulish..., fig. 7,8 .

I16 Le premier fragment de la Graufesenque provient des fouilles de 1966 et pourrait, d apres l'usage qui est fait des imbrications, être daté du règne de Néron, au plus tôl. le tesson de Rode\%, conserví au musée Fenaille, est un inédit de la collection Ylermet, datable par son decor (médaillons de sautoirs stylises, du regne de claude.

117 op. cit., pl. 116, 3.

118 Pour le premier, le choix est guidé par le sens habituel 'pointe en haut des imbrications, pour le second, il est determiní par la presence de sujets figurés (des aigles? 
le choix de leur orientation ${ }^{119}$. Ainsi, nous avons la preuve qu'il a existé des valves inférieures à feuillure. sans avoir la certitude que toutes les pièces pareillement moulurées avaient le même emploi.

Le second groupe, constitué de deux fragments, sort visiblement d'un mème atelier et l'identification de la forme demeure hypothétique : il s'agit, en effet, de matrices destinées a la partie inférieure de vases globuleux et l'hésitation est possible entre les ovoüdes et les cruches. C"est la taille des échantillons ${ }^{120}$ et. surtout, l'organisation du décor en registres soigneusement différenciés qui incitent à supposer, au moins à titre dhypothese, qu'il peut s'agir dr valves de moules à lagènes. Il existe certes, d'assez gros vases ovoüdes à décor moulé, mais ils sont rares; d'autre part, quelle que soit la dimension du support, aurune des frises ornementales que je connais de LIBER'TYS ou de ses successeurs idlentifiés ou non ne m'a semblé, jusqu'a présent, présenter de façon habituelle ce systeme de métopes et de bordures, sur des formes globuleuses simples.

Le premier tesson (fig. 15. 1; appartient à une grande matrice a la paroi épaisse, grossierement tronconique et dont le protil se rétrécit vers le bas ; le bord supérieur el la base de l'objet font également défaut. Le décor (figr. 15, 16 , limité à sa partie inférieure par une ligne de $S$ est composé de métopes séparées verticalement par des cordons tiretés. Je répertoire est réduit : une rosette inédite a sept larges pétales; les séparations : tiretées ou en fin zigzag (horizontal); un fleuron. lui aussi inconnu par ailleurs; deux masques. L'un, très petit, représente de profil un visage d'homme, au front plissé. au ne\% court, à la bouche lippue ; le cràne est ì demi chauve, la barbe annelée : il peut s'agir d'un silline. Le second. comparable au précédent par le type du visagee. est. en revanche, trés gros : il en subsiste une barbe peignée en boucles verticales. une parlie de la bouche épaisse et l'extrémité de la checelure frisée. taillée à la hauleur du lobe de l'oreille. Le pelit masque ressemble étonnamment à celui qu'ulilise le groupe de LIBERTVS'21. mais je n'ai pas eu entre les mains d'érhantillons de ce dernier assez bien conservés pour permettre une comparaison exacte. Le gros silène. inconnu d'oswald. semble ne pouvoir ètre que la réplique. en plus grand. d'un poincon attesté par les rases de IANVARIVS' I. à Rheinzahern. A en juger d'après la qualité du modelé et la laille. la malrice de Lezoux pourrait correspondre à un état antérieur d'un lype repris ensuite dans l'list ${ }^{122}$. IATVARIVS. passe pour aroir débuté sous Antonin ${ }^{123}$. notre poinçon. dans cetle hypothese. serait rontemporain ou antérieur.

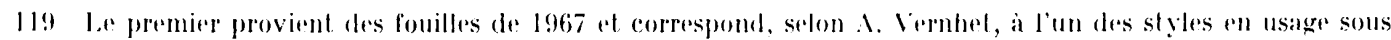
Tibere. Ie second, un incid de la collection Hermet, porte un rincean a fenilles palmees, comme on en voit, à la Graufesenque, surtout sous taude et Xéron. On peut ajouter a ces ichantillons celui que reproduit Hermet fpl. 116., si, toutefois, il ne sagrit pas d'une reconstitution thérique. Le premier tesson est orne de festons, habites de lievres au repos, disposes obliquement : les orienter la tête vers le haut - ce qui paraìt le plus frequent dans ce genre de decor. fait de la matrice une valve inferieure. Quant au second, labsence de sujet figure dans le rinceau, interdit de se prononcer sur la place de la valve.

1:20 I. diam. max. pour la partie conservere du premier tesson est deja de 26 en a lexterieur ; lo diam. interieur du second, an niveau du hord est de $21 \mathrm{~cm}$. Pour les emands ovoüles monles, voir, par exemple, un vase de PaTER.

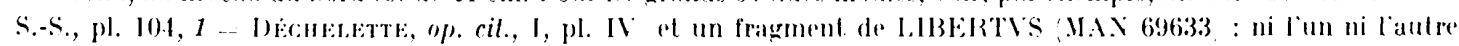
de ces modeles, decoris, l'un de melopes, lautre d'une frise continue, ne comporte de regristres aussi soigneusement differenciés les deux zones du vase de l.IBERTYS ne sont pas nellement separés ; ancun n'est borde d’un motif à repelition.

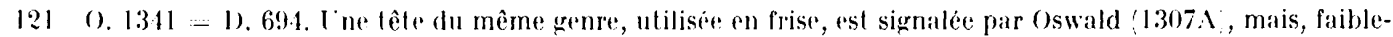
ment imprimée vers le menton, elle a té consideree comme un modele distinct de 13.11. I.es masques de notre bordure sont lantot forterment, tantot inçalement empreints et paraissent selon le cas, el alors qu'il s'agit du même poincon, proches, à s'y meprendre, du modele de I.IBERTVS-BVTRI0, ou, au contrajre, semblables a $0.1307 \mathrm{~A}$.

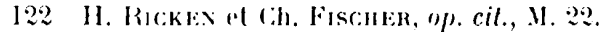

123 P. Kanxits:u, op. cit., p. 252-254. 
Le second tesson (fig. 15. 2), appartient au bord supérieur d'un moule du même grenre. mais un peu plus petit. Le décor (fig. 15, 2b), à deux zones, est composé de métopes, semblables a celles de l'autre fragment el d'une frise de pelits masques barbus. longeant le hord, a la maniere des oves. Outre ce silène, les deux lypes de séparations. la rosette, le fleuron - communs aux deux moules *; cet échantillon porte de nouveaux motifs : une petile sirene, de face; les restes d'un masque féminin, de profil ; la partie supérieure d'un énorme visage de femme, représenté de face, avec des oreilles maladroitement figrurées et une chevelure ondulée en courtes ragues. Ia composition paraît la mème dans les deux décors : le potier utilise tantôt de très largres métopes, occupées, sur nos fragments, par un gros masque, tantòt d'étroits bandeaux rerticaux, meublés, de haut en bas, d'une frise composite. dans laquelle entrent masques, figures mythologiques, fleurons... L'atelier est par ailleurs connu, par l'habitude qu'a le décorateur d'utiliser la tête de silene en guise d'ove. F. Oswald a daté certains bols 1). 37 ainsi ornés du règne de Trajan. Cependant, le réper-
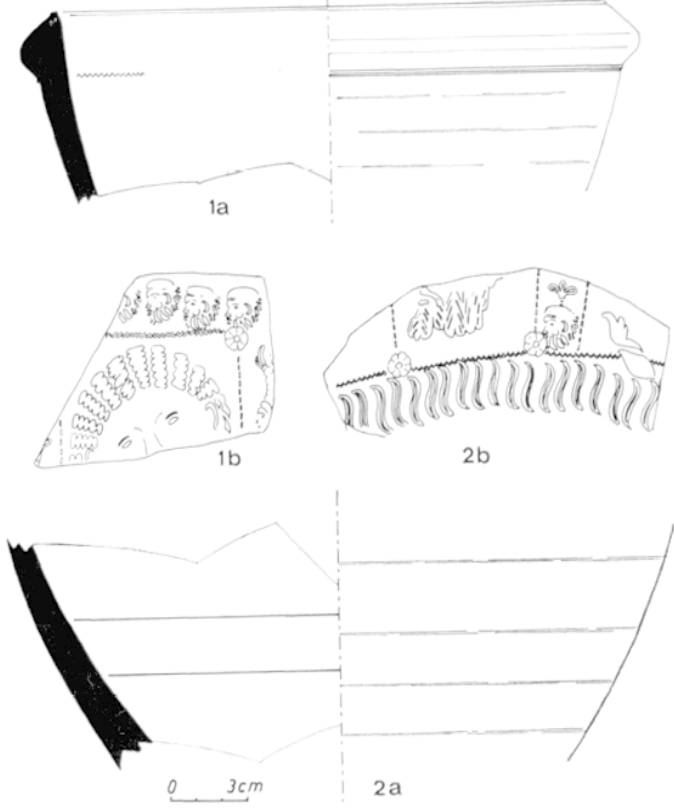

15) Voules de Ieroux potier aux masques, premiere moitié du $11^{\mathrm{e}} \mathrm{s} . ?$ ?: 1 a : coupe at profil du bord d'un moule de lagrene ou de gros ovoüde; 1 b : décor du même / non inverse ; 2 a : coupe ef profil d'un fragment de moule de lagene ou de gros ovoüde; $2 b$ : decor du même non inversé. toire des quelques fragmentsde moules el de vases conservés à Saint-Germain invite à situer, plus prudemment, cette fabrication dans la première moitié du ${ }_{\mathrm{I}} \mathrm{e}$ siècle ${ }^{124}$. Ia qualité des deux grands moules, en lout cas ...- pâte fine, ocre rosé, micacée, peu cuite pour l'un, imparfaitement oxydée, pour l'autre - est compatible avec cette chronologie ${ }^{125}$. Et les caracteres du style s'accommodent aussi d'une telle datation : la taille monstrueuse de l'un des masques, il est vrai, n'a actuellement d'analogues connus que parmi les appliques utilisées un peu plus tard sur les vases ovoïdes lisses, ce type féminin, qui n’a pas été signalé a Lezoux, se dérobe en outre à la comparaison, et l'on ne connaît, du gros silène, que de petites répliques dans l'Lst ; toutefois, des compositions du même grenre apparaissent sur des produits de I.IBERTVS' et BVTRIO. (n voit, en eflet, alterner sur des formes I). 30 ou 37 d'étroits panneaux, occupés, souvent, par des caryatides, et de larges métopes, meublées tantòt de personnagres, lantòt de masques énormes'26. A l'argument fourni par cette similitude technique, s'ajoute celui qui constitue la parenté étroite de détails des deux répertoires ${ }^{127}$. Il n'y a donc pas d'invraisemblance à placer le potier aux masques dans le milieu où travaillait l'équipe de LIBERTVS.

Il n’échappe pas que l'identification de ces moules, peut, si elle est confirmée par des vases, présenter un interèt particulier. Les deux premiers tessons analysés apportent un supplément d'information pour une période où la lagène est connue, non seulement dans

12.4 Motifs identifies : moules (D. 37, numérotés pour une part M.\.69635, cavalier : cf. O. 243 ; homme nu :

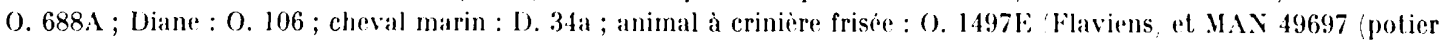

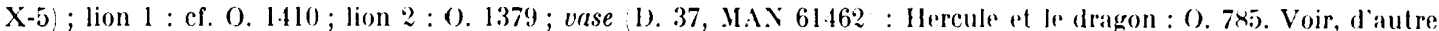
part, a propos du caractere précoce des fins traits ondulés all n $^{\mathrm{e}}$ siecle, (i. Sispsox, op. cil., p. 152.

125 Elle est, par son aspect, tout a fait comparable à celle de moules de I,IBEITVS, BVTRIO, SECV.NDINVS

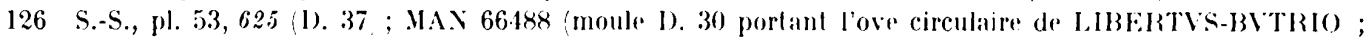
IIAN 49694 (I). 37, style de BVTRIO .

127 (cf. outre le petit masque, la sirine (0. 863 et 86.31$)$. 


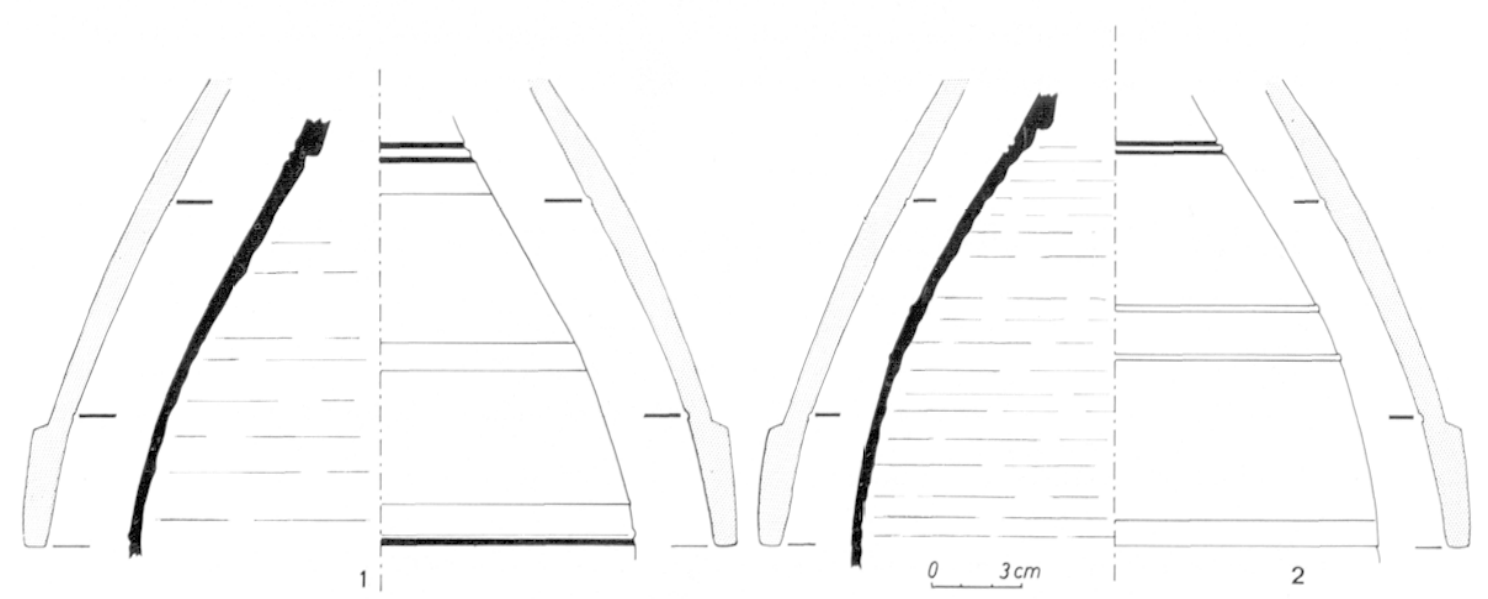

16 Profils compares. 1 : galbe interieur du moule de cil'sss coupe en gris, et partie supérieure de la lagène Mirabilis; 2 : galbe interieur du moule de C:ATVSSA et partie superieure de la lagene SABINI .I /Rodez. (Les deux profils de lagenes sont etablis d'apres des releves d'A. Vernhel. - On voit, par comparaison avec des vases sortis de moules legerement plus petits que celui de Levoux, $a$ : les varialions de ditail dans le profil de la lagéne H. $1 \bar{j}, b$ : la similitude globale du galbe interieur de notre valve ot du galbe extérieur des deux lagenes appartenant au même type.

le Sud, mais aussi à Lezoux. En revanche, les derniers, s'ils ne relevent pas, simplement, d'une variété originale de vases ovoüdes, pourraient témoigner de la permanence, ou de la résurgence, des lagènes dans la génération, voire le groupe, de LIBERTVS ou BVTRIO et expliquer, pour une part, la création insolite de liATSSSA.

Il ressort de cette brève étude que l'on ne peut encore raisonner à propos de la cruche de notre potier que par analogie ; l'existence durable des lagènes sigillées à Lezoux est démontrée, nous l'avons vu, mais sporadiquement et par de petits et rares échantillons; aussi la typologie locale de cette forme demeure-t-elle incertaine. Nous avons noté, en effet, que la production de modèles à valve inféricure décorée était probable, sans être assurée, et constaté, d'autre part, que la forme de certains bords de moules, à date haute, était compatible arec une technologie comparable à celle de la Craufesenque. Il s'agit là d'olservations de portée limitée, qui ne permettent pas de dessiner une évolution typologique et, dans le cas précis de la matrice de CATVSSA, les comparaisons les plus pertinentes ne peuvent se faire qu'avec un matériel étranger.

Le profil de la pièce apparente incontestablement cette dernière à des exemplaires de la Graufesenque (fig. 16), qui relivent du type is d'Itermet et comportent, habituellement, deux valves décorées: la forme presque exactement tronconique de l'intérieur du moule est en tous points semblable à la ligne extérieure de la grande lagène, de la lagène .Mirabilis et d'autres aussi, et sensiblement différente, en revanche de la coupe piriforme - due a l'abaissement de la panse de la cruche de Baginton, ressortissant au type 62 de I)échelette ${ }^{128}$. Cependant, nous n'avons actuellement aucune certitude concernant la permanence de liens nécessaires entre la typologrie de la valve supérieure et celle de la partie inférieure du vase : le choix d'un profil piriforme ou ovoïde el d'un mode de décoration est-il déterminé par le type général du récipient, indépendamment de la chrono-

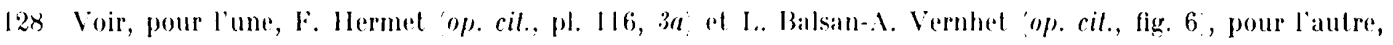
J. A. Stanfield (Romano-gaulish..., fig. 7 ). 
logie? La forme est-elle, comme il semble, liée à la date de fabrication, que le bas de la panse soit ou ne soit pas orné? Certaines particularités sont-elles, enfin, fonction à la fois de la date et du modèle, si l'on estime, comme on l'a fait déjà pour l'atelier Rutène, que les rases piriformes sans frise inférieure sont caractéristiques d'une haute époque ${ }^{129}$ ? Aucune de ces questions, dans sa généralité, n’a reçu de réponse. Pourtant, les échantillons de la Graufesenque -.- les mieux connus prêtent à un certain nombre de remarques : la lagène $H$. 15 et la cruche $\mathbf{D}$. 62 sont attestées, l'une et l'autre, assez tot ${ }^{130}$; quelle que soit la date, le bas des panses entièrement décorées est, d'ordinaire, faiblement galbé; ;orsqu'on possède des fragments suffisants des décors inférieur el supérieur d'un mème exemplaire, on constate que le profil de la partie haute est tronconique, peu bombé. la forme générale, ovoïde ou bitronconique ${ }^{131}$. Les exemples recensés tendent ainsi a montrer qu'on a fabriqué vers 30/50 deux cruches de formes différentes, diversement ornées : la première, ovoïde et entièrement décorée, appartient à un type qui s'est maintenu au moins jusqu'à la fin du ier siècle. En revanche, les exemplaires datés de la seconde - piriforme - sont si rares, ou rarement publiés, qu'il n'est possible encore ni de fixer la durée du modele, ni d'en montrer la constance. ou la variété, voire le caractère purement accidentel (du moins en terre sigillée) ${ }^{132}$. Il a existé probablement des contaminations, ou des hésitations, pour les modes d'ornementation : une lagène de Rodez, ovoüde, très galbée du bas, et particulièrement lourde et disgracieuse. est lisse en sa moitié inférieure ${ }^{133}$, mais il n'en demeure pas moins que la plupart des échantillons connus associent à un profil tronconique supérieur, une partie basse peu galbée et que les cruches décorées en leur zone inférieure constituent une écrasante majorité. Il reste aussi que cet embryon de typologie concerne le matériel

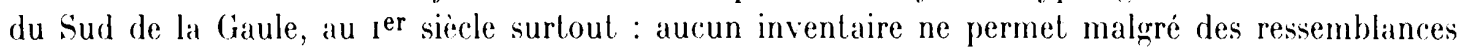
indubitables, d'affirmer que les mèmes principes sont applicables rigoureusement aux vases du Centre, produits au $\mathrm{II}^{\mathrm{e}}$ siècle. De plus, nous ne disposons d'aucun argument technologique qui autorise à préjuger du couplagre de notre valve avec un moule complémentaire : on a découvert à la Graufesenque des matrices pourvues ou dépourvues de dispositifs d'emboitement ou de pose ${ }^{134}$; certains exemples, à Hontans ${ }^{135}$, de valves tant inférieures que supérieures paraissent privées de mode d'articulation. Il semble, pour le moment que des procédés différents aient été utilisés aux mêmes fins (peut-être selon la date) dans le Sud : on peut supposer, provisoirement. qu'il en fut de même ailleurs et que le jumelage des valves s'accommoda de variations dans la forme des bords.

129 J. A. STavilite, ibid., p. 168 et G. Mary, op. cil., p. 177-178. I.es objections presentees par G. Mary relativement aux indices chronologiques proposés par J. A. Stanfield portent sur l'usage des oves non sur la decoration de la valve infericure ou la forme generale de la cruche. A la Graufesenque, actuellement, on ne connaît pas de lagenes piriformes autres que de haule époque.

130 C.f. n. 106.

131 (if. J. A. STaxfei.s, ibid., fig. 8 lagène Hartlip; pl. XXI lagine de Rodez signée SABIXIM, en fait beaucoup plus trapue ; pl. XXIII lagene de Windisch'; pl. XXIV lagene de l.ondres; ; I.. BAI.SA.N ef A. VERxnET, op. cit. Lagine . Virabilis et grande lagene; inediles: collection Rey, nos 4 at 5 ; lagene de Iagamas (Hérault'. Il existe, par ailleurs, à la Graufesengue au moins trois types de petites lagenes, à valve inferieure decorée ot de forme plus ou moins hemisphérique; clles sont antérieures a la période de splendeur ou contemporaines de celle-ci.

132 La lagene de Baginton est la seule piece entière de ce type qui ait eté publier. Il n'y a pas lieu, en effet, de retenir l'exemplaire de Londres etudie en 1929 par le même J. A. Stanfield linusual forms of Terra sigillata, dans Archaenlogical Journal, 86, p. 114-117 et considere comme une lagene J). 62 decored de haut en bas. Iat restilution de la forme paraissait à l'auteur lui-même, quelques années plus tard, trés douteuse Komano-gaulish..., p. 16r, surtout apres la revelation du type 15 d'Hermet: il ne subsiste que deux petits fragments de l'objet.

133 Ce modèle inédit m'a été aimablement communiqué par L. Balsan et $\mathrm{A}$. Vernhel.

134 Certains fragments de valves ne conservent pas de traces de feuillures. I)autre part, il existe, au moins un autre type de moule se prêtant au couplage : les foulles récentes, en particulier celles de la campagne 1971 , ont prouvé l'existence, indubitable, de matrices comportant une levre plate, soignneusement équarrie, à l'époque tibérienne.

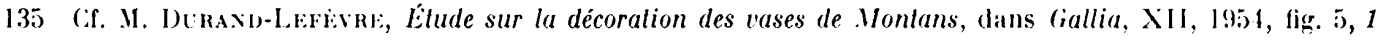
et 6 . 
Ainsi, à s'en tenir à la forme générale, les fragments signés par CATVSSA n'ont d'analogues hien connus que parmi les matrices ou vases du I $^{\text {er }}$ siècle, dans le Sud de la Gaule, et principalement à la Ciraufesenque, où ce type de demi-moule est habituellement associé à un moule complémentaire - tronconique ou légèrement galbé ..., lui-mème décoré, mais la solution de continuité que l'on constate actuellement, à l'intérieur de l'atelier de Lezoux, entre les exemplaires identifiés et, d'un atelier à l'autre, entre les derniers échantillons datés de la Graufesenque et la matrice de CATVSSA, interdit de présumer de la portée de ces observations at de parler avec pertinence et précision d'une pérennité de la forme de la lagène.

En ce qui touche l'équilibre du décor, on peut voir, si l'on compare entre elles différentes cruches II. $15^{\mathbf{1 3 6}}$, que la hauteur de la partie ornée de la valve supérieure représente souvent entre $2 / 3$ et $3 / 4$ du diametre maximum du récipient et comporte, en général de 2 à 4 registres. Ein revanche, le rapport différent dans lequel se trouvent les deux parties de la panse du type piriforne D. 62 (alors que les dimensions maximales sont analogues) entraine, naturellement, une modification de la relation entre la hauteur el le diamètre du décor : dans le cas du vase de Baginton, elle atteint un peu plus des $4 / 5$, pour une frise répartie en trois zones. La partie conservée du moule de CATTVSSA permet d'établir un rapport proche de 2/3, en considérant la limite probable de la seconde rangée de métopes. Cette remarque n'a de valeur qu'indicative, puisque le haut de la matrice fait défaut, mais le décor des lagènes s'arrète au-dessous du col et l'étranglement de la valve de Lezoux, au niveau de la cassure semble exclure un rétrécissement beaucoup plus prononcé de l'orifice supérieur du moule et, par conséquent, un développement considérable de l'ornementation au-delà du dernier registre conservé. Il y a donc quelques vraisemblances en faveur d'une similitude de proportions entre ce décor et ceux de lagènes H. 15.

Ainsi, il est vrai que nous ne sommes pas encore en mesure d'établir l'existence d'un rapport nécessaire et durable entre l'équilibre du volume el l'économie de la frise qui orne (es cruches, mais du moins pouvons-nous souligner que la distribution des motifs sur le flane de la valve de Lezoux et la forme du profil intérieur du moule se prêtent l'une et l'autre à des comparaisons intéressantes avec un certain nombre de lagènes ovoüdes de la Graufesenque.

Le choix des thèmes décoratifs, enfin, compte tenu de la disposition en bandes, habituelle sur les lagènes, appelle peu de commentaires. L'alternance des groupes de personnages et des frises végétales est chose courante, soit sur la mème valve, soit dans l'ensemble d'une ornementation, et le bas du moule supérieur est souvent occupé par des rinceaux ${ }^{137}$. On remarquera, surtout, en la circonstance. l'absence d'oves. L'usage de cet ornement est très fréquent, sinon général, sur les cruches du ier siècle à la Graufesenque, et la valve supérieure attribuex à TITOS, ainsi que les fragments de Saint-Germain datés de la seconde moitié du $\mathrm{I}^{\mathrm{er}}$ siècle en comportent également. J'ignore, faute d'une documentation abondante pour le $\mathrm{II}^{\mathrm{e}}$ siècle, si la suppression de cette frise dans le décor de CATVSSA doit être considérée comme un fait occasionnel ou comme l'expression d'une règle commune sous le règne d'Hardrien ou un peu plus tard.

Colette Bémont.

136 J'ai pris ces mesures pour les lageines Hartlip, de Windisch, du Causse-Noir, de Rodez, la lagène Mirabilis, la grande lagine.

137 Cif. la grande lagene, les lagenes de Baginton, de Rodez, du Causse-Noir, de Windisch.

$N$. B. Les clichés des fig. 2 a 6 ont èté aimabiement fournis par la photothèque du Nusée des $A$ ntiquitís nationales. 